\title{
Paul Sabourin
}

sociologue, département de sociologie, Université de Montréal

(2005)

\section{"Médiateurs et médiations sociales constitutives de l'epistemè de la connaissance économique au Québec dans la première moitié du XXe siècle"}

Un document produit en version numérique par Jean-Marie Tremblay, bénévole, professeur de sociologie au Cégep de Chicoutimi

Courriel: jean-marie tremblay@uqac.ca

Site web pédagogique : http://www.uqac.ca/jmt-sociologue/

Dans le cadre de la collection: "Les classiques des sciences sociales"

Site web: http://classiques.uqac.ca/

Une collection développée en collaboration avec la Bibliothèque

Paul-Émile-Boulet de l'Université du Québec à Chicoutimi Site web: http://bibliotheque.uqac.ca/ 
Cette édition électronique a été réalisée par Jean-Marie Tremblay, bénévole, professeur de sociologie au Cégep de Chicoutimi à partir de :

Paul Sabourin, "Médiateurs et médiations sociales constitutives de l'epistemè de la connaissance économique au Québec dans la première moitié du XXe siècle".

Un article publié dans la revue Sociologie et sociétés, vol. 37, no 2, automne 2005, pp. 119-152. Montréal: Les Presses de l'Université de Montréal.

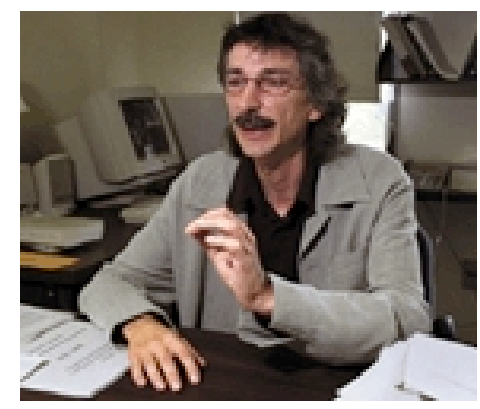

M. Paul Sabourin est professeur de sociologie au département de sociologie de l'Université de Montréal.

[Autorisation formelle de diffuser cette œuvre accordée le 30 janvier 2004 par l'auteur.]

Courriel : paul.sabourin@umontreal.ca

Polices de caractères utilisée :

Pour le texte: Times New Roman, 14 points.

Pour les citations : Times New Roman, 12 points.

Pour les notes de bas de page : Times New Roman, 12 points.

Édition électronique réalisée avec le traitement de textes Microsoft Word 2004 pour Macintosh.

Mise en page sur papier format : LETTRE (US letter), 8.5" x 11")

Édition numérique réalisée le 12 décembre 2006 à Chicoutimi, Ville de Saguenay, province de Québec, Canada. 


\section{Paul Sabourin}

"Médiateurs et médiations sociales constitutives de l'epistemè de la connaissance économique au Québec dans la première moitié du XXe siècle"

\section{SOCIOLOGIE ET SOCIÉTÉS}

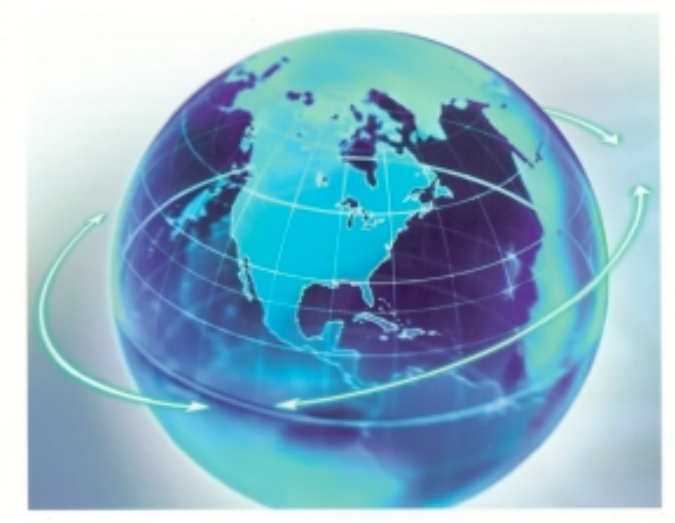

Le Québec et l'internationalisation des sciences sociales

Les Presses de P'Université de Montréal

Un article publié dans la revue Sociologie et sociétés, vol. 37, no 2, automne 2005, pp. 119-152. Montréal: Les Presses de l'Université de Montréal. 


\section{Table des matières}

$\underline{\text { Introduction }}$

1. La « brèche » ou l'hétérogénéité de la connaissance économique et son statut paradoxal dans la pensée économique

1.1. La genèse hétérodoxe de la pensée économique au Québec

2. Propriétés des savoirs critiques de l'économique

2.1. Médiations sociales et valeurs économiques : les thèses de H. Arendt

2.2. La localisation sociale de la pensée au fondement de l'appropriation des médiations de la connaissance

3. Entre transcendance religieuse et universalisme économique

3.1. L'espace social de la pensée économique

3.2. Le dispositif de l'économique universel : les fondements cognitifs du développement

3.2.1. La Revue économique canadienne : travail de connaissance, travail de diffusion

3.3. Édouard Montpetit, médiateur

4. L'actualité économique : le travail de déconstruction de l'économique comme universalisme

4.1. Le travail intellectuel de la «science » économique appliquée ou la genèse de la connaissance administrative

4.2. La valeur d'usage comme lieu de médiation conceptuelle et le refus de l'autonomisation de l'économique

CONCLUSION

Résumé

Bibliographie 


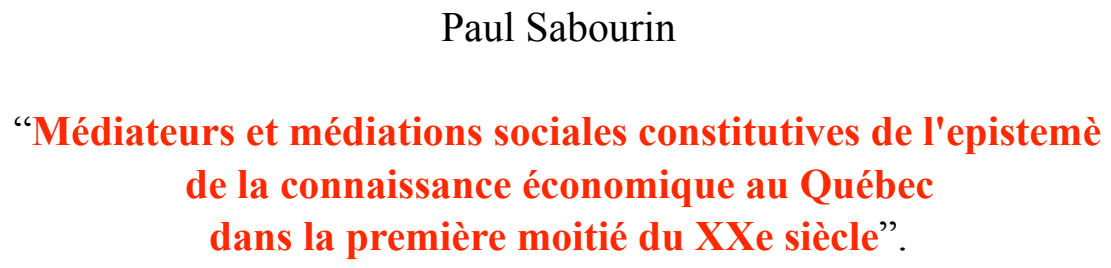

Un article publié dans la revue Sociologie et sociétés, vol. 37, no 2, automne 2005, pp. 119-152. Montréal: Les Presses de l'Université de Montréal.

« ... ma conviction est que la pensée elle-même naît d'événements de l'expérience vécue et doit leur demeurer liée comme aux seuls guides propres à l'orienter. »

H. Arendt

La crise de la culture, 1954

\section{Introduction}

Le travail de décantation et d'épuration en sciences sociales tend à gommer l'hétérogénéité des œuvres pour une lecture du passé confortant la cohérence d'une perspective présente (Berthelot, 1998 ; Ramognino, 2000, p. 154). Dans le cas des sciences sociales au Québec, parler en termes d'épuration et de décantation serait employer un euphémisme pour qualifier le rapport entre savoirs « traditionnels » et savoirs « modernes » : il ne s'agit pas tant d'épuration ou de décantation que d'invalidation des savoirs traditionnels par les modernes, et aujourd'hui, par ceux qui se définissent comme post-modernes. Au lendemain de la Seconde Guerre mondiale, sur la base de la constitution d'un savoir " moderne » en science économique comme en sciences sociales, l'invalidation de ce savoir a pris le sens de l'opposition de la « science » à l'idéologie traditionnelle « catholique ». De nos jours, où ces prétentions à une scientificité acquise des sciences économiques et sociales ont été radicalement remises en question et apparais- 
sent même puériles, l'interprétation post-moderne reconduit cette invalidation sur la base de l'opposition entre une pensée pluraliste et une pensée monolithique, c'est-à-dire totalisante, gommant tout autant cette hétérogénéité des oeuvres. Il faut dire que dans la pensée occidentale, il a fallu plusieurs décennies et des penseurs remarquables, telle $\mathrm{H}$. Arendt, pour proposer une analyse plus lucide des prétentions des « modernes » :

Chez Marx, comme dans le cas de nombreux grands auteurs du siècle dernier (XIXe), un ton apparemment enjoué, provoquant et paradoxal, dissimule l'embarras d'avoir à traiter des phénomènes nouveaux dans les termes d'une vieille tradition sans la charpente conceptuelle de laquelle la moindre pensée semblait impossible. (Arendt, 1972, p. 37)

Dans ses révoltes contre les savoirs traditionnels et ses déclarations universalisantes, la sociologie contemporaine au Québec ne présentet-elle pas de nouveaux contenus en recourant à d'anciens cadres conceptuels ? Nous avancerons à la suite de H. Arendt que les conditions de la pensée « moderne »nécessitent une objectivation et une prise en compte des médiations dont procèdent de façon implicite les sciences sociales afin que soient substantiellement renouvelés leurs cadres de connaissance.

Dans une première partie théorique, nous verrons que l'analyse de cette auteure met au jour le rôle central des médiations sociales de la pensée permettant d'appréhender l'hétérogénéité des oeuvres comme traces de processus de connaissance : traces d'« expériences de pensées ». Nous étudierons celles-ci sous l'angle de leur construction sociale dans le cadre d'une sociologie de la connaissance afin de montrer que la réduction de l'activité intellectuelle à une position idéologique n'est pas satisfaisante parce qu'elle efface le travail qui s'effectue en prenant comme point d'appui le relevé des déclarations normatives du chercheur à des moments de son travail qui figurent comme synthèse 
de « sa pensée " ${ }^{1}$. Or, comme nous allons le voir, même quand les intellectuels prétendent arriver à cette cohérence, la synthèse logique de la pensée n'existe précisément pas parce que l'organisation de leur travail répond à plusieurs logiques sociales mises en jeu dans un travail intellectuel qui est pluriel.

Notre démarche vise à proposer une voie d'analyse de ce travail à partir des notions de médiation et de localisation sociale (Halbwachs, 1952). Le cheminement consiste à appréhender la pluralité des expériences sociales de pensées dues aux médiations tout en esquissant une morphologie sociale de celles-ci qui se différencie des conceptions des catégories de l'histoire intellectuelle classique : " La pensée », « Le penseur », "La cohérence logique ». Pour nous, il s'agit de donner corps à la conceptualisation de la distribution sociale du savoir (Munck, 1999) à travers des formes sociales de connaissance (Houle, 1979) issues de l'activité en réseaux qui sont l'échelle et le lieu de l'effectivité de la pensée (Descombes, 1996).

Pour ce faire, la genèse de la pensée économique "moderne », et particulièrement le cas de figure que représente sa genèse hétérodoxe ${ }^{2}$ au Québec ${ }^{3}$, est un terrain des plus fertiles pour saisir ce caractère hétérogène du travail de connaissance qui s'accentue lors d'une période de transition. L'émergence de ce savoir met en jeu des confrontations entre formes de connaissance, types de travail intellectuel,

1 Pour prendre les termes de M. Granovetter (1985), il y a une production d'un modèle "sursocialisé » de la vie sociale lorsque les inférences du chercheur induisent le contenu des interactions sociales à partir des seuls constats de proximité entre personnes (adhésion à un mouvement politique, fréquentation d'une personne, présence dans un même espace conçu comme une totalité sociale qui surdétermine, etc.).

2 Nous allons définir un peu plus loin dans cet article notre usage des termes économique, économie, orthodoxe et hétérodoxe.

3 Avant les années 1960, l'espace national du Québec correspond à l'espace social du Canada français. 
qui provoquent des mouvements de distanciation ${ }^{4}$, résultats de ces confrontations. Ces mouvements de distanciation suscitent à leur tour des tentatives de remaniement ou de réorganisation implicites ou explicites de ces savoirs, comme dans le cas des perspectives critiques de l'économie politique au XIXe siècle, ceci afin de constituer un espace de pensée, c'est-à-dire une épistémè. La transition ne se définit plus alors comme un simple passage, mais comme une temporalité spécifique où apparaissent des traces dans l'analyse du fondement et de la relativité des formes sociales vécues notamment à travers ces moments et lieux de confrontation entre formes de connaissance.

Du point de vue théorique, nous allons donc nous inspirer de l'analyse remarquable de cette transition faite par H. Arendt. Son approche critique, tant des savoirs traditionnels que modernes, dont celui de l'économique, permet de problématiser le travail intellectuel non seulement comme connaissance du monde, mais aussi comme rapport au monde. Suivant cette auteure, l'apport de ces grands penseurs du XIXe n'est plus donc à rechercher dans une cohérence idéologique, mais bien dans leurs « contradictions fondamentales et flagrantes ». En ce sens, elle propose une nouvelle approche théorique des processus de la connaissance à la lumière de l'expérience de la modernité. Cet espace entre formes de connaissance, qu'elle désigne comme une «brè-

4 L'engagement normatif, c'est-à-dire la position idéologique, ne saurait résumer le travail de connaissance comme le souligne Élias (1993, pp. 10-11) : « Les concepts d'engagement et de distanciation seraient donc fort inadéquats en tant qu'outils de pensée si on leur associait la représentation de deux tendances humaines indépendantes l'une de l'autre. Ils ne se rapportent pas à deux groupes séparés de phénomènes psychiques. Employés comme catégories, ce sont en tout cas des notions limites. En règle générale, nous observons des êtres humains et leurs manifestations - par exemple, des types de langage, de pensée et autres activités - dont certains sont marqués par une assez forte distanciation alors que d'autres se caractérisent par un assez fort engagement. Entre ces deux pôles s'étend un continuum et c'est lui qui constitue le véritable problème. » 
che 5 » entre savoirs « traditionnels » et « modernes », constitue une situation sociale particulière à ces « grands » penseurs du XIXe siècle qui se généralisera à tous au XXe siècle. Cette situation permet des opérations de pensée à travers différents savoirs dont l'origine sociale est différenciée : les fameuses déclarations de retournement, de remise sur ces pieds des savoirs religieux et philosophique, tout comme les affirmations d'un rapport critique à l'économie politique. Pour $\mathrm{H}$. Arendt, ces médiations sociales de la connaissance l'amènent à conclure à une dégradation des valeurs traditionnelles et plus généralement de la connaissance dans la modernité sous la forme d'une idéologie relativiste qui traverse le sens commun comme les sciences sociales.

Pour notre part, cette dégradation relativiste ne représente qu'une des voies possibles qu'ouvre la nouvelle morphologie sociale de la connaissance propre à la modernité. Nous montrerons que ces opérations, parce qu'elles sont à la mesure des médiations internationales qui vont présider à l'élaboration d'une connaissance de l'économie au Québec, peuvent donner lieu à l'identification de ses dimensions sociales et ouvrir la possibilité d'un savoir dont le statut peut se poser dès lors comme général plutôt que dans les anciens cadres de pensée du local et de l'universel.

Mais pourquoi cette hétérogénéité des savoirs a-t-elle commencé à être pensée au XIXe siècle, notamment dans le domaine de la pensée économique, et seulement dans les approches critiques de l'économie politique?

5 L'image de la brèche par H. Arendt (1972) est évocatrice du fait que les normes idéologiques qui s'affrontent provoquent une distanciation de celles-ci. Nous pensons, comme l'avançait $\mathrm{H}$. Arendt, que cette «brèche » entre les savoirs du passé et ceux du futur a toujours existé, conditions existentielles de l'être humain dirait la philosophe, mais de notre point de vue, elle relève de la matérialité sociale de la pensée humaine qui devient perçue, comme celle-ci l'affirme, dans la modernité. 


\section{La «brèche » ou l'hétérogénéité de la connaissance économique et son statut paradoxal dans la pensée économique}

$\underline{\text { Retour à la table des matières }}$

Dans un article intitulé « Transition et pensée économique », Gilles Dostaler relève la constitution hétérogène du savoir économique et son statut paradoxal chez les penseurs de l'économie, même lors de l'émergence du « monde contemporain » :

Les fondateurs de l'économie politique classique vivent en effet la plus spectaculaire des transitions parmi celles que nous avons décrites, la naissance du "monde contemporain », dans toutes ses dimensions. Ils expliquent comment hâter cette transition, étant de ce fait très conscients du lien entre le fonctionnement économique et les institutions sociales ; pour paraphraser Marx, ils expliquent comment les rapports de production féodaux bloquent le développement des forces productives. Et pourtant comme les philosophes grecs, les scolastiques ou les mercantilistes, ils nient la transition en tant que telle, et plus catégoriquement encore que leurs prédécesseurs. On peut dire que l'économie politique se constitue comme discipline autonome en niant la transition, c'est-à-dire en postulant des lois naturelles de fonctionnement de l'économie. (Dostaler, 1988, p. 26)

La pensée économique dans ces périodes de transition manifeste une constitution duale, elle est à la fois l'élaboration d'un savoir adapté à la situation historique, ce que nous dénommons aujourd'hui le discours d'économie politique, et à la fois un savoir énonçant des lois naturelles et universelles du genre humain, associé à l'économique, c'est- 
à-dire à ce qui deviendra la science économique ${ }^{6}$ orthodoxe contemporaine. Ce dernier savoir se conçoit comme activité essentielle sans qu'elle se donne aucun statut du point de vue de son épistémè : la naturalité des comportements humains devrait en soi imposer son existence au-delà des discours et des cultures par son caractère d'universalisme économique.

À l'autonomisation de l'économique comme système correspond l'autonomisation de la "science économique »comme savoir (Rioux, 1984, p. 3). Si ce constat est juste, la question qui se pose est de comprendre pourquoi persiste aussi longtemps une vision totalisante et unitaire de ce savoir ; nous dirions aujourd'hui une " pensée unique ». Dans quelles conditions cette constitution hétérogène des savoirs économiques va-t-elle être finalement appréhendée ? Les constats sur les perspectives hétérodoxes et critiques qui refusent partiellement ou totalement l'autonomisation de la science économique peuvent nous

6 Nous nous fions ici pour définir l'économique comme savoir savant aux travaux de Michel Beaud et Gilles Dostaler (Beaud et Dostaler, 1993, p. 129) qui constatent cette dualité du discours économique et le rôle fondamental de l'orthodoxie de l'homo economicus dans l'univers des discours en science économique : « Cette orthodoxie, qui s'enracine dans certains aspects de l'économie classique, mais a été engendrée par la révolution marginaliste, privilégie l'homo economicus, et donc une rationalité générale portant sur quelques choix élémentaires, le marché, l'équilibre, l'optimum. Elle ne prétend pas rendre compte directement du monde concret, mais elle s'impose comme système de référence à l'ensemble des économistes, universitaires notamment. Le paradoxe est qu'aucun des classiques, aucun des pères de la révolution marginaliste, aucun des grands économistes du vingtième siècle qui ont contribué au développement de l'analyse néoclassique, aucun n'a limité sa pensée ni son oeuvre à ce travail d'élaboration théorique dans ce cadre à l'irréalisme convenu. Il n'empêche : l'indestructible construction néoclassique continue à dominer le débat théorique et l'enseignement en économie. " Pour cette raison, il faut se méfier des définitions des dictionnaires généraux qui, par la nature du travail qu'ils impliquent, produisent, au sens de F. Dumont, des syncrétismes en superposant dans une définition de l'économie politique des phrases tirées d'oeuvres orthodoxes, hétérodoxes, d'économie politique classique, marginaliste, etc., pour les confondre dans un seul terme confus et vague d'économie politique. 
éclairer sur cette question et nous permettre de mieux cerner la spécificité de la connaissance économique au Québec.

Il faudra attendre les travaux de Schumpeter et de Keynes, à l'écart de la tradition dominante (Dostaler, 1988, p. 33), pour voir apparaître la dimension temporelle de l'économie appréhendée en termes de conjoncture historique, c'est-à-dire dans une conception du temps circonscrit en court et moyen terme ${ }^{7}$. Mais cette entorse à l'orthodoxie économique demeure partielle. Ce n'est qu'après la Seconde Guerre mondiale et quand est posée la situation économique des pays du « tiers-monde » que des économistes formuleront des théories de la transition proposant des analyses du développement et de la dépendance.

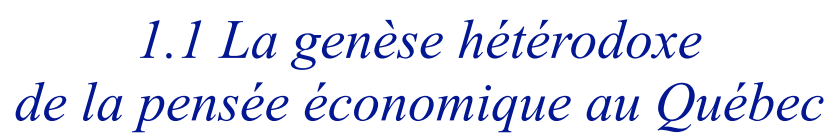

$\underline{\text { Retour à la table des matières }}$

C'est à ce dernier mouvement de développement de la connaissance économique que nous pouvons le mieux rattacher la dynamique de la connaissance économique qui va émerger au Québec bien qu'elle s'élabore dès le début du XXe siècle. Comment expliquer cette genèse hétérodoxe de la connaissance économique au Québec, d'une connaissance plurielle de l'économie, c'est-à-dire qui dans son évolution produira une pluralité plus grande qu'ailleurs de conceptions de l'économie : « d'écoles épistémologiquement repérables » pour reprendre le constat de Gilles Paquet (Paquet, $1985 ; 2000^{8}$ ).

7 Comme le souligne Dostaler (1988), Keynes a formulé sa vision de la temporalité de la "gestion de la conjoncture » dans une boutade montrant bien l'horizon de sa pensée : «À long terme, nous serons tous morts 》 (ibid, p. 33). La dimension temporelle est introduite mais avec une réduction drastique.

8 Voir à ce sujet l'analyse de Paquet (2000) de l'écart entre la pratique de l'économie politique au Québec et de la science économique, et de celle de la différenciation entre les économistes des facultés d'administration et de sciences économiques. 
On pourrait répondre à cette question en soulignant la situation de domination dans laquelle se développent l'économie et la connaissance économique au Québec, situation qui viendrait en déterminer sa structuration originale comme pour les pays du « tiers-monde ». Cette relation n'est pas fausse, mais n'est pas celle d'une détermination causale. Si on se réfère de différentes façons à ces rapports de domination économique, ceux-ci sont appropriés par un travail de connaissance chez les premiers économistes canadiens-français.

Par appropriation sociale, nous entendons ici le travail de connaissance ${ }^{9}$ qui inscrit les êtres dans le « réel perçu », qui apparaît alors multiple, voire fragmenté. Nous allons essayer de montrer la pertinence pour les travaux futurs de rendre compte de la complexité de cette appropriation sociale résultante de ce travail à travers les médiations sociales et les médiateurs, personnes et réseaux sociaux qui ont contribué à développer la pensée économique au début du XXe siècle. Nos points d'ancrage pour esquisser ces réseaux de connaissance seront les oeuvres et les activités d'Édouard Montpetit, participant de la première génération d'économistes des HEC 10 et d'Esdras Minville, " disciple » de Montpetit, et donc de la " seconde génération ». Ces travaux servent de lieux de références et de discussions pour la majorité des premiers économistes québécois. Le premier point d'ancrage nous permettra d'examiner la confrontation entre des rapports au monde relevant de la transcendance religieuse et de l'universalisme économique qui déterminera un espace résiduel à la pensée économique. " une brèche » entre les savoirs traditionnel et moderne.

Édouard Montpetit se posera en médiateur entre le savoir des premiers économistes étrangers venus enseigner aux HEC et les idéolo-

9 Si la connaissance est une mise en forme de l'expérience selon des règles implicites ou explicites, dans le cas de la connaissance savante, cette mise en forme est aussi le résultat d'un travail du langage sur le langage (ex. : le journalisme comme langage qui met en forme un langage premier, celui du sens commun). Voir à ce sujet Houle (1979).

10 HEC est l'acronyme de Hautes études commerciales de Montréal. 
gues canadiens-français ${ }^{11}$ afin de faire admettre que le problème économique est déterminant de l'avenir national. Ce rôle de médiateur est tributaire d'un réseau intellectuel franco-québécois que Gérard Fabre a mis au jour, en montrant le caractère nodal des activités de Montpetit et son rôle de catalyseur à partir de différentes sources d'emprunt, dans le développement du savoir universitaire au Québec : «Montpetit ne peut cacher cette profonde ambivalence qui l'autorise à valoriser aussi bien "les méthodes françaises" que la "science américaine" » (Fabre, 2005, p. 75). Une idéologie fondée sur le principe du « juste milieu » anime ce réseau : " cette idéologie relève aussi d'un parti pris sur le monde social et peut souvent s'analyser comme un alibi qui permet d'évacuer les vrais problèmes » (Fabre, 2005, p. 62). En effet, comme nous le verrons, Montpetit se cantonnera dans une conception de principe du « juste milieu » conciliant et résorbant la « brèche » issue des antagonismes et des incompatibilités entre les savoirs économique et religieux.

Le second point d'ancrage, les travaux d'Esdras Minville, nous amènera à analyser le déplacement de cette conception doctrinaire du savoir vers un savoir empirique de l'économie : une connaissance économique appliquée ouverte sur l'observation. Nous chercherons à faire état du travail de distanciation du religieux comme de l'économique de Minville rouvrant cette « brèche » entre les savoirs qui va entraîner le développement de l'activité de recherche.

Dans l'espace de cet article, prenant cette ligne d'analyse, nous ne visons pas à dresser un portrait d'ensemble des activités intellectuelles pendant ces 50 premières années de la pensée économique au Québec. Nous nous contenterons d'étayer sa pertinence afin de situer cette genèse par rapport aux courants critiques de l'économique. Notre démarche empirique ne sera pas habituelle. Elle croisera des considérations souvent définies comme niveaux différents d'analyse. la socialisation des personnes, les réseaux, l'espace institutionnel, les discours écono-

11 Il s'agit de l'élite cléricale de Montréal, Mg, Bruchési et les milieux nationalistes conservateurs : les personnes gravitant autour d'Henri Bourassa, particulièrement le politicien Armand Lavergne. Pierre Harvey (1994, p. 156) montre bien le caractère non monolithique du milieu nationaliste : des fervents nationalistes, tel J.-X. Perreault, seront aussi des promoteurs des HEC. 
miques, tous ces éléments seront conjugués afin de faire état du travail de connaissance sous l'angle des médiations et de leur articulation à travers les relations sociales qui composent et localisent la pensée.

\section{Propriétés des savoirs critiques de l'économique 12}

$\underline{\text { Retour à la table des matières }}$

Il est bien connu que c'est dans le courant critique de l'économie politique de Marx et des marxistes par la suite que l'on trouve dès le XIXe siècle les premières constructions de la transition. Au centre du modèle économique du Capital est introduite une dynamique sociale : les rapports de domination capitaliste entre classes qui marquent l'originalité de l'appropriation marxienne des discours de la science économique (Granger, 1967, p. 1023). D'autres dimensions de la morphologie sociale des économies sont esquissées. La localisation spatiale des économies, résumée notamment dans la distinction des différents modes de production, et même celle de la connaissance économique, puisque " les catégories de l'économie politique sont historiquement déterminées ». Est donc établi un premier lien chez Marx entre la structuration de la connaissance et la structuration d'une vie sociale où l'économique domine comme catégorie de connaissance.

Dans une perspective marxiste, Luckas tentera d'expliciter ce rapport entre connaissance et économie : l'économie comme forme sociale de connaissance (Houle, 1987) aboutissant à l'analyse de la conscience de classe. La notion de médiation apparaît dans le langage marxiste afin de poser le problème de l'hétérogénéité des valeurs hu-

12 Le terme économique renvoie à la forme de connaissance produite par l'objectivation matérielle des faits humains grâce à des savoirs techniques, telle la comptabilité, et des savoirs savants : la science économique. Le terme d'économie vise l'ensemble du phénomène humain concret que l'on peut découper dans son aspect sociologique : activité sociale de production, de circulation, de distribution et de consommation, l'économie consiste aussi en un aspect des autres pratiques sociales (Godelier, 1984). 
maines et de résoudre ce problème dans les termes de la dichotomie entre valeur d'usage et valeur d'échange. Celle-ci prendra différents sens : allant de la dégradation de la valeur à des conceptualisations plus complexes en termes d'objectivation, de réification, d'aliénation.

Ce sont ces questions qu'aborde H. Arendt dans La crise de la culture, où elle tente de sauver l'entreprise philosophique face à cette révolte des penseurs du XIXe siècle contre les savoirs traditionnels qui, à travers les "sauts et renversements " des cadres conceptuels anciens, cherchent à invalider la pertinence du savoir philosophique et à arracher ses notions, pour les redéfinir comme affaire du commerce entre les hommes : lieu « réel » de la valorisation.

\subsection{Médiations sociales et valeurs économiques : les thèses de H. Arendt}

\section{Retour à la table des matières}

S'interroger sur les médiations consiste donc à s'interroger sur les éléments de la connaissance qui peuvent être considérés comme conservés ou altérés, et dans quelles conditions (Hennion, 1993, p. 222224). Si ces médiations sont historiques et biographiques, la connaissance qu'elles mettent en jeu est constitutive d'un rapport au monde spécifique. Voilà la première thèse que soutient Arendt contre le relativisme.

Plutôt que de confiner ce constat à une critique des oeuvres, sa seconde thèse est qu'il est nécessaire d'objectiver ces médiations comme point de départ de sa propre pensée. Autrement dit, d'un point de vue méthodologique, comment pouvons-nous faire état de telle transition dans la connaissance s'il y a transmutation du sens ? Nous retrouvons ici le problème ancien du cercle herméneutique dans ces différentes déclinaisons (Molino, 1985) que tente d'aménager H. Arendt devant ce que sont les conditions de la pensée moderne et la transmission des savoirs traditionnels : " Notre héritage n'est précédé d'aucun testa- 
ment 13 » (René Char cité par H. Arendt). Dans « la tradition et l'âge moderne », H. Arendt nous propose une analyse de la genèse de la pensée contemporaine à partir des oeuvres de Marx, Kierkegaard et Nietzsche. Son objet n'est pas d'expliquer spécifiquement la genèse de la pensée économique, mais d'assurer plutôt une continuité de sens du savoir philosophique : d'une philosophie politique qui est menacée par l'émergence du savoir économique comme nouveau rapport au monde. Ce faisant, elle attaque de plein fouet la volonté de révolte de ces penseurs qui déclarent que « la philosophie et la vérité ne se trouvent pas en dehors des affaires des hommes et de leur monde commun, mais précisément en eux » (Arendt, 1972, p. 28) et qui ne situe pas leur travail de pensée dans ce monde. Ainsi, la seconde thèse d'Arendt avance que ces contradictions relèvent des médiations entre savoirs, médiations subies plutôt qu'objectivées et maîtrisées par ces penseurs qui se croient faussement en rupture radicale avec les cadres conceptuels anciens. C'est ce que montre l'utilisation de la notion de valeur chez Marx :

Sa distinction entre « valeur d'usage » et « valeur d'échange » reflète la distinction entre les choses en tant qu'utilisées et produites par les hommes et leur valeur dans la société ; et son insistance sur l'authenticité plus grande de la valeur d'usage, sa description fréquente de l'apparition de la valeur d'échange comme une sorte de péché originel au commencement de la production marchande reflète sa reconnaissance impuissante et pour ainsi dire aveugle de l'inexorabilité d'une imminente « dévaluation de toutes les valeurs ». La naissance des sciences sociales peut être située au moment où toutes les choses, les « idées » aussi bien que les objets matériels, furent mises en équation avec les valeurs, de sorte que chaque chose tenait son existence de la société et y était reliée, le bonum et le malum non moins que les objets tangibles. Dans le débat sur la question de savoir si c'est le capital ou le travail qui est la source des valeurs, on oublie généralement qu'à aucune époque antérieure à la Révolution industrielle naissante on ne professa que les valeurs, et non pas les choses, sont le résultat

13 Autrement dit, la tradition n'existe que sous la forme de sa réactualisation dans les catégories présentes, elle ne peut donc être envisagée dans sa transmission comme une inculcation, une influence, etc. Cette découverte de la co-construction du sens des textes remet en question certains discours historiques qui proposent une interprétation sans médiation des textes passés (voir à ce sujet Halbwachs, 1968, p. 50). 
de la capacité productive de l'homme, pas plus qu'on ne rattacha tout ce qui existe à la société et non à l'homme « pris isolément ». (Arendt, 1972, p. 48)

Malgré la transmutation ${ }^{14}$ de la valeur, le discours moderne des savoirs économiques reconduit l'ancien cadre de pensée de l'unicité du monde à travers celui d'une théorie unitaire de la valeur et des prix. Plus encore selon Arendt, ces discours visent à hâter cette transition dans la mesure où ils subordonnent la pluralité des valeurs émanant de la confrontation des formes de connaissance à l'économique (Brochu, 2002) 15 : les valeurs redéfinies dans les discours d'économie politique, orthodoxe comme hétérodoxe, font que celles-ci sont devenues interchangeables, commensurables et fonctionnelles dans un monde qui n'apparaît plus que comme celui de l'administration des êtres et des choses. £idée de dégradation des valeurs du point de vue philosophique n'est pas totalement évacuée non plus. Ainsi, chez Marx, nous dit Arendt, la valeur d'échange vient dégrader la valeur d'usage qui, elle, est naturalisée comme le nouveau socle transcendant et implicite de toutes les valeurs. Ce n'est que depuis quelques années que cet économisme de Marx à propos de la valeur d'usage a été correctement identifié dans le Capital bien qu'il ait affirmé dans d'autres écrits que l'utilité des choses était relative aux sociétés (Denis, 1980 ; Habermas, 1985) 16.

Une des premières déductions possibles de cette approche de la connaissance de $\mathrm{H}$. Arendt est de nous indiquer où chez les premiers penseurs de l'économie au Québec, qui par ailleurs voulaient plutôt assurer la continuité des savoirs traditionnels, va se poser le nœud gordien de la transformation de la connaissance. La question de la va-

14 Transmutation signifie « un changement radical d'une substance en une autre ». En physique, c'est la transformation d'un élément simple en un autre par modification du noyau atomique (Encyclopédie Universalis, " transmutation »).

15 Voir à propos de la notion de subordination économique Paul Brochu (2002).

16 Voir à ce sujet les travaux de Marie-Claude Rose (2001), qui reconstruit la valeur d'usage et d'échange comme médiation de la circulation et de la consommation. 
leur d'usage sera effectivement le point tournant du schème conceptuel comme nous le verrons dans les travaux de Minville. Elle est le lieu de l'adhésion ou du refus de l'autonomisation de l'économique.

Mais, ces opérations de pensée faites pour rendre compte d'une réalité perçue comme bouleversée par la Révolution française et la Révolution industrielle, rendues possibles par les conditions nouvelles propres à la modernité, seront aussi celles qui dans le cas de Marx l'amènera à identifier les dimensions sociales des économies, leurs variations de forme dans le temps et l'espace, mais aussi les variations de langage pour parler de ces réalités. Si H. Arendt souligne que ces propriétés de l'humain socialisé seront totalisées dans la notion idéologique de société, il n'en reste pas moins que son analyse indique que c'est seulement à travers l'étude des médiations historiques et biographiques que l'on peut remettre en cause cette totalisation fondée sur une conception métaphysique plutôt que concrète de la pensée et de son objet. Or, malgré la critique d'Arendt, il faut noter les acquis du travail de Marx par sa tentative de mettre en rapport connaissance et économie. L'économie politique est en relation avec l'expérience dans la mesure où ses catégories sont historiquement déterminées, c'est-àdire situées dans un état de développement des rapports sociaux, ce qui est déterminant de l'usage qu'il en fait dans ses discours d'économie politique. En revanche, ce mouvement de prise en compte des médiations est partiel dans son oeuvre et neutralisé par le recours aux catégories économiques (ex. : la valeur d'usage). Pourtant, la prise en compte des médiations devrait constituer un des éléments fondamentaux d'une démarche de connaissance savante comme l'avance $\mathrm{H}$. Arendt, mais ce mouvement doit être constant en science comme dans les « sciences sociales ", si l'on veut les distinguer d'une production idéologique. Pour traduire dans les termes de l'épistémologie contemporaine, la science se caractérise notamment par un travail de remise 
en question du « rapport d'une forme à un contenu suscité à partir d'une expérience renouvelée » (Granger, 1991) ${ }^{17}$.

La rupture que nous avons constatée dans la connaissance économique au Québec montre bien que cette transmission problématique se pose d'une façon similaire, aboutissant à la prégnance des ruptures en l'absence de règles de cumul de la connaissance. À défaut d'une normativité scientifique (Houle et Ramognino, 1993, p. 8) qui opérerait une réciprocité des perspectives explicite, c'est la normativité sociale dominante implicite de la fabrication du savoir selon les règles médiatiques qui s'impose en sciences sociales 18 .

Pour rendre opératoire notre démarche, il faut considérer que la confrontation de ces formes de connaissance chez les premiers penseurs de l'économie est faite non seulement d'un travail de " l'oeuvre» par l'expérimentation de différentes formes de discours (essai, description statistique, monographie, etc.) qui déconstruit et reconstruit la connaissance, mais aussi dans la rédaction et l'édition liées aux revues qu'ils produisent, aux réseaux où ils discutent leurs connaissances, aux institutions auxquelles Us participent. Chez Montpetit, la localisation sociale de son discours donne lieu à la juxtaposition plutôt qu'à l'arti-

17 Nous nous référons ici à une des distinctions que fait G.-G. Granger entre la science comme travail et l'idéologie comme production spontanée : « ... l'idéologie apparaît comme production spontanée, par opposition à la science, qui est travail. Ceci ne signifie pas que l'expression des formations idéologiques ne puisse être le résultat d'une poḯsis et d'une élaboration secondaire interne qui peut les constituer en appareils complexes. Mais on peut bien dire que l'idéologie ne travaille pas dans la mesure où elle ne remet plus en question le rapport d'une forme à un contenu suscité à partir d'une expérience renouvelée n'est nous qui soulignons). Elle se donne ce rapport une fois pour toutes et ne fait éventuellement varier que la forme qu'elle isole. Tout en prétendant décrire et régir l'expérience, elle échappe essentiellement à son contrôle »(Granger, 1991, p. 95). Ce qu'identifie H. Arendt à partir de l'analogie de la brèche puis du modèle d'analyse des contradictions des penseurs renvoie à la genèse de ce travail de connaissance en sciences sociales.

18 Ces normativités sont le pseudo-scientisme (Granger, 1991) et la normativité de l'édition littéraire intervenant dans la production des sciences sociales. 
culation d'un langage économique et d'un langage religieux : celui du mal économique et du culte de l'argent.

\subsection{La localisation sociale de la pensée au fondement \\ de l'appropriation des médiations de la connaissance}

\section{$\underline{\text { Retour à la table des matières }}$}

Dès 1925, M. Halbwachs constate la pluralité des cadres sociaux de la mémoire individuelle (Sabourin, 1997). Il étudie les modalités des processus de connaissance à travers cette pluralité de notions issues de l'appartenance d'une personne à des groupes sociaux différenciés. Il montre qu'à l'échelle individuelle, la mémoire sociale opère à partir de raisonnements sociaux. Ces raisonnements sont la mise en oeuvre de notions servant à assembler les souvenirs. Elles sont issues des relations sociales et forment des zones sociales dans la mémoire : une localisation sociale constitue un mode d'organisation sociocognitif. En prenant l'exemple de sa propre mémoire, Halbwachs décrit bien ce travail de connaissance comme le résultat d'une dynamique d'assimilation et d'accommodation de cette organisation en rapport à l'expérience. Il est possible dans l'activité de penser, de parcourir sa mémoire en se déplaçant à travers les points de vue qui la forment. La formation de ces points de vue ne renvoie pas uniquement aux relations de coprésence chez M. Halbwachs, mais aussi aux activités de lecture et d'écriture : au travail de connaissance qui est ainsi localisé socialement. Du point de vue analytique, les médiations de la connaissance sont sociales parce que résultant d'activités sociales. Elles le sont aussi dans un sens second, par l'usage social que l'on peut faire des productions de cette connaissance médiatisée, lequel est localisé à travers les relations socio-symboliques constitutives du travail de connaissance. 
Afin de délimiter la localisation sociale de ces penseurs ${ }^{19}$, en nous référant au constat de Fernand Dumont (Dumont, Montminy et Hamelin, 1971, p. 1) sur les idéologies de cette époque, nous dirons qu'à la différence de la majorité des idéologues « qui ne sont pas à même de saisir les phénomènes nouveaux que sont l'industrialisation, l'urbanisation de la société canadienne-française ", les premiers penseurs de l'économie seront localisés par une éducation issue d'un rapport de connaissance fondée sur la transcendance du religieux et une scolarisation fondée sur l'universalisme économique. Dès lors, la question est de savoir comment ils parviendront, à travers leur travail intellectuel respectif, à se mouvoir dans cette « brèche » ouverte par la confrontation de ces formes de connaissance.

\section{Entre transcendance religieuse et universalisme économique}

$\underline{\text { Retour à la table des matières }}$

Dès le XXIe siècle, des écrits canadiens-français traiteront de la question économique. Ils prendront la forme d'essais, mais aussi d'un roman. On retient particulièrement les publications d'Étienne Parent et surtout celles d'Errol Bouchette, dont les idées vont constituer un plaidoyer pour la prise en compte des transformations économiques étatsuniennes. Ces idées seront reprises systématiquement et retravaillées par les premiers penseurs de l'économie (Paradis, 1982). Échappe à ce portrait l'œuvre originale d'un des précurseurs de la sociologie au Québec : le père de Léon Gérin, Antoine Gérin-Lajoie (Sabourin, 1994). Bibliothécaire au parlement d'Ottawa, il a accès à des livres étrangers. Il prend connaissance des Ouvriers européens de Frédéric Leplay et transpose sa méthode pour l'étude du milieu des Bois-Francs au Québec où il se rend chaque été à sa ferme (Lemire, 1980). Il entreprend d'écrire une monographie afin de montrer l'existence « d'un

19 On comprendra ici que, proposant une sociologie de la connaissance plutôt qu'une histoire, nous utilisons le terme de localisation sociale plutôt que celui de la description du contexte souvent utilisé en histoire. Nous nous sommes déjà expliqué ailleurs sur la non-pertinence de l'emploi du terme de contexte en sociologie (Sabourin, 1993). 
esprit de développement économique » des petites villes de cette région. Ce développement constituerait une réponse à l'émigration massive des Canadiens français en dehors de la ruralité (dans les villes au Québec, au Canada et aux États-Unis). Des éléments monographiques subsistent dans ce qui prendra d'abord la forme d'un " récit de la vie réelle ». Sous les injonctions de son éditeur, il en fera un roman : Jean Rivard le défricheur et l'économiste est la mise en scène, à travers ce personnage, de la complémentarité de la vie agricole et industrielle. Antoine Gérin-Lajoie prend la défense de l'industrialisation rurale qui n'apparaît ni contradictoire au développement de l'agriculture ni contradictoire à la morale catholique. L'auteur met en scène une discussion entre l'artisan de ce développement et le curé de la paroisse pour mieux expliciter les conditions dans lesquelles une accumulation économique individuelle est justifiée de par son usage familial (Gérin-Lajoie, 1993). Il souhaite aussi l'ouverture du milieu rural à l'immigration étrangère comme un apport nécessaire à cette industrialisation rurale. Ce sont là des idées originales pour l'époque. Si la description technique du développement d'une ferme agricole est relativement précise pour ce genre de document, il n'en est pas de même pour ce qui est des petites industries. Le sujet est davantage examiné du point de vue de ses conséquences sur la vie des villages et de ses implications morales.

À notre connaissance, on ne trouvera aucune référence à cet ouvrage dans les écrits des premiers penseurs de l'économie 20 même si le rapport problématique entre religion et économie s'y trouve posé. Ce roman connut pourtant une très large diffusion (16 éditions) au Québec de même qu'en France dans le journal Le Monde (à Paris en 1900) sous forme de feuilletons. Les premiers penseurs de l'économie se référeront à cette période de l'économie capitaliste d'avant 1890 comme étant révolue, comme un capitalisme à l'état premier : « atomiste » dira par la suite Jean-Charles Falardeau.

20 Il n'y a pas de référence à cette œuvre chez les premiers économistes. Édouard Montpetit cite Léon Gérin-Lajoie, que l'on nomme aujourd'hui Léon Gérin, et non pas Antoine, son père, lors de ses conférences à l'université Mount Allison en 1928 publiées dans la Revue trimestrielle canadienne. Peut-être la confusion est-elle due au fait que certains parlent comme Victor Barbeau de Léon Gérin en le dénommant uniquement par son nom de famille « Gérin-Lajoie ». 


\section{3-1 L'espace social de la pensée économique}

\section{$\underline{\text { Retour à la table des matières }}$}

À la fin du XIXe siècle, plusieurs processus sociaux vont concourir à la naissance des HEC : l'émergence d'une presse d'affaires francophone qui n'est pas sans lien avec la dissociation des francophones de la Chambre de commerce de Montréal pour créer leur propre chambre de commerce ${ }^{21}$. Une hypothèse intéressante est avancée par l'historien P. Harvey qui fait remonter l'intérêt pour les questions économiques des francophones à Montréal et la création des HEC aux constats des inégalités de conditions des francophones et des anglophones durant l'épidémie de 1881. En fait, le mouvement pour la création des Hautes études commerciales (1907), soutenu par la Chambre de commerce de Montréal, se réalise dans la période de Révolution industrielle du Québec (1900-1929) qui correspond à l'établissement d'industries coloniales surtout britanniques dans les grandes villes du Québec comme Montréal. Un espace institutionnel pour la pensée économique est inauguré en 1911, mais il demeurera précaire. Le véritable processus qui établira cette connaissance dans la société québécoise reste à venir ; processus de légitimation de la problématique de ce discours puis de définition de son champ spécifique : "Parmi les différentes institutions qui diffusent un savoir à caractère économique, l'École des hautes études commerciales de Montréal est sans aucun

21 La création de journaux économiques francophones, Le moniteur du commerce (1881), puis Le prix courant (1886), vont être des lieux de diffusion des débats qui vont aviver la constitution d'un réseau social spécifique aux gens d'affaires francophones, lequel s'institutionnalisera dans la création, en 1886, de la Chambre de commerce canadienne-française de Montréal. On associe à ces journaux la promotion de certaines thèses du libéralisme économique. 
doute celle qui a donné l'impulsion nécessaire au développement d'une discipline économique » (Fournier, J., 2005, p. 389) 22.

Les membres de la Chambre de commerce de Montréal vont être mobilisés pour assurer la création des HEC et le recrutement du premier corps professoral. Par leurs réseaux d'affaires, issus notamment de leur participation aux expositions universelles et celles de l'Empire britannique, des experts les conseilleront dans le recrutement des premiers économistes étrangers qui assureront la légitimité universitaire de l'école. Cette légitimité universitaire au Québec ne peut avoir comme fondement que les médiations institutionnelles étrangères, c'est ce qu'apprendra durement Édouard Montpetit dont les premiers

22 Cet espace institutionnel restera longtemps précaire. L'École des HEC est, en vertu de la loi, autonome et laïque. Plusieurs tentatives seront faites, par les autorités religieuses qui contrôlent l'enseignement universitaire, pour rattacher les HEC à la structure de l'enseignement supérieur existante. Elles réussiront pendant une certaine période : de 1914 à 1956. L'affiliation des HEC, école neutre en 1914, à l'Université de Montréal, initiée par Mg' Bruchési auprès du premier ministre Lomer Gouin, confère un statut catholique à l'école et en ferme ainsi l'entrée aux protestants. En 1926, avec la constitution du deuxième régime administratif, le gouvernement dissout la corporation autonome de l'école et crée une Corporation des écoles techniques et professionnelles relevant directement du Secrétariat de la province. Sous ce régime, l'école se retrouve encore affiliée à l'Université de Montréal qui sanctionne le régime pédagogique. Voir à ce sujet l'histoire des HEC de Harvey (1994, 2002). Pour situer l'enseignement des HEC par rapport aux autres institutions, voir Fournier, 2005. 
enseignements de l'économique seront remis en question ${ }^{23}$. Le recrutement du premier directeur impliquera plutôt Honoré Gervais, membre de la Chambre de commerce de Montréal et le professeur Blondel, des Hautes études commerciales de Paris. Ce recrutement s'effectuera après une investigation des candidats possibles en France, en Belgique et en Suisse. Mg, Bruchési intervient aussi dans cette nomination en appuyant une candidature, montrant ainsi que lors de la création des HEC les autorités religieuses ne s'opposent pas à la constitution de la première école laïque du Québec (Harvey, 1994, p. 115).

Parmi trois candidatures envisagées, c'est celle de A.-J. Debray qui sera retenue pour combler le poste de directeur de l'école. Le nouveau directeur interviendra d'une façon déterminante sur l'espace institutionnel de la pensée économique en train de se constituer, puisant dans sa propre formation et jouant de son réseau de relations dans les écoles de commerce européennes ${ }^{24}$. L'enseignement de l'économie politique au Québec commencera donc dans une école supérieure qui vise la formation à la pratique commerciale plutôt qu'une formation à la « science » du capital.

23 Édouard Montpetit se fera remarquer pour une conférence qu'il donne sur l'histoire de l'économie politique à la Société de l'économie sociale de l'Université Laval. Un compte rendu élogieux en sera fait dans le Bulletin de la Chambre de commerce. Cette même université créera un cours d'économie politique en 1907, qui sera assigné à Édouard Montpetit. Dans ses Souvenirs, Montpetit rappelle les termes très durs utilisés par un journaliste du Canadien pour mettre en doute sa compétence à enseigner sans « des études profondes » en économie politique et une légitimité étrangère : « En France, cette nomination ferait sourire » (Harvey, 2002). Cette critique l'amènera à écrire une lettre au premier ministre Lomer Gouin où il propose sa candidature comme professeur aux HEC et demande une aide financière pour entreprendre des études d'économie politique et de géographie économique en Europe.

24 D'après l'évaluation de F. Harvey, ancien directeur des HEC, « il s'agit d'un programme de facture plutôt européenne... » (ibid., p. 182). 


\subsection{Le dispositif de l'économique universel : les fondements cognitifs du développement}

\section{$\underline{\text { Retour à la table des matières }}$}

Comment faire admettre que le problème national des Canadiens français est un problème économique ? La création de l'école des HEC peut être vue comme une stratégie pédagogique pour établir d'abord l'existence du problème économique qui viendra justifier la nécessité de sa connaissance. La mission de l'école est de penser le développement et transmettre les outils de cette pensée : l'économique est désormais conçu par les discours de l'école comme une force civilisatrice universelle et, en ce sens, il devient la valeur déterminante de l'accès aux autres valeurs (culturelles, spirituelles, etc.). Ce qui est nouveau, ce n'est pas tant de traiter de l'économie que de traiter de l'économie en termes économiques.

Ce dispositif comprend une bibliothèque économique et un musée commercial en plus des programmes d'enseignement. Au conseil d'administration de l'école tel que le mentionne le Livres des minutes des HEC de 1909, un comité réunit des informations sur les écoles supérieures de commerce et fera des visites en Europe et aux États-Unis pour déterminer les enseignements. L'accent est mis par le directeur sur les méthodes d'insertion des étudiants dans la vie économique : le bureau commercial, les visites industrielles, les projections cinématographiques. Dès 1908, A.-J. Debray annonce la création de la Revue économique canadienne dont le premier numéro paraîtra en 1911. Tout se met en place pour faire admettre l'évidence du mouvement économique universel et tenter d'intégrer aux catégories d'entendement des Canadiens français les outils cognitifs permettant de penser dans les termes de développement : la lecture de l'événement plutôt que de l'avènement, la mesure statistique qui fonde une lecture du progrès économique ou de la régression, l'appréhension de l'espace « mondial » et des réalités « universelles » en opposition au local.

Cette première revue de l'école dirigée par A.-J. Debray connaîtra une fin abrupte en 1914, et Debray sera congédié de la direction des 
HEC ${ }^{25}$. Ce congédiement en 1916 est provoqué par la vive réaction des idéologues canadiens-français à cette vision universelle de l'économique. En 1925, l'Association des diplômés des HEC recrée la revue, cette fois intitulée L'actualité économique, revue qui existe aujourd'hui encore sous ce nom.

L'analyse de la constitution de ces revues nous donne accès aux différents types de travail intellectuel qui vont permettre de constituer et de légitimer un espace pour la pensée économique au Québec.

\subsubsection{La Revue économique canadienne : travail de connaissance, travail de diffusion}

$\underline{\text { Retour à la table des matières }}$

La première parution énonce les objectifs de la revue et le statut du travail effectué par ses rédacteurs : "C'est par l'étude de toutes les questions économiques et leur vulgarisation, par la publication de faits économiques mondiaux, par l'exposé de renseignements utiles sur la production universelle ...»(HEC-REC, 1911, p. 1). Cet accent sur le référent universel peut nous faire sourire aujourd'hui, considérant que notre époque s'octroie le fait de la mondialisation économique. À partir de ce référent, le champ de la revue est très large et s'inspire du sous-titre de la revue européenne Le moniteur des intérêts matériels : "Tout ce qui est d'intérêt général, hormis la politique ». Au moment où sont écrites ces lignes, ce qui justifie cet accent, c'est l'œuvre nationale parce que le sort de la classe laborieuse est lié au développe-

25 L'historien Rumilly attribue ce départ à la réaction aux mœurs du directeur et à l'indiscipline des étudiants de l'école, tandis que F. Harvey note les affrontements relatés dans le Livre des minutes entre celui-ci et le président du bureau Isaie Préfontaine à propos de la gestion de l'école et des dépenses personnelles de Debray. Les réactions qui corroborent le fait que la vision du contenu de la revue est problématique pour la direction figurent dans les modalités de cessation de la revue. Face au déficit de la revue, A.-J. Debray offre de continuer la revue sans subvention de l'école; il reçoit comme réponse « qu'il ne serait pas opportun d'accorder une telle autorisation à moins que le nom de l'École disparaisse de la revue et que la rédaction se fasse hors de l'édifice de l'école » (Livre des minutes, 1914). 
ment économique international. La ligne directrice de la revue est de faire admettre que le problème national est un problème économique.

Le contenu de la revue est divisé en quatre sections : "À l'École des hautes études commerciales », "Revue des faits économiques », « Revue bibliographique » et une section de deux ou trois articles sur des questions économiques. Une cinquième section sera ajoutée la deuxième année : «Jurisprudence commerciale ». Ce travail s'appuie sur une bibliothèque qui compte pour un dixième du budget total de il école, montrant ainsi son importance. Dès la première année, la revue met en évidence la richesse de cette bibliothèque qui renferme plus d'un millier de monographies, sans compter une centaine de revues économiques et autres documents (HEC-REC : 1, 7, p. 233).

L'essentiel du travail intellectuel de la revue consiste à produire des résumés-synthèses 26 à partir de différents matériaux : statistiques économiques, articles de périodiques, livres sur la question économique. Il ne s'agit pas d'une revue strictement d'économie politique, elle est composée en partie d'un travail journalistique associé à ce que nous appellerions aujourd'hui la presse d'affaires. La connaissance des théories économiques est abordée directement dans la section " Revue bibliographique ». La méthode "scientifique » privilégiée de l'économie politique, les statistiques, donne lieu à des rubriques dans plusieurs sections et des articles par A.-J. Debray hors section : ce sera la voie de transmission de cette lecture du monde en termes d'universalisme économique.

La première section "À l'École des hautes études commerciales » compile des informations sur les activités d'enseignement de l'école (conférences, excursions pédagogiques, etc.) mais aussi des renseignements sur l'état de l'enseignement commercial au Québec, au Canada et à l'étranger (Barcelone, Paris, etc.). Ce domaine est sous la responsabilité du directeur et des membres du bureau administratif de

26 Voici la description générale de ce travail dans la revue : « S'attacher à étudier, à analyser et à exposer d'une façon complète et méthodique les grands mouvements économiques du monde en vue d'en tirer le meilleur parti au point de vue national ... » (HEC-REC, 1, 1, p. 2). 
l'école qui iront aussi visiter les écoles commerciales américaines au cours du développement des programmes.

La deuxième section de la revue affirme l'économique comme un fait. Son caractère à première vue encyclopédique, " tout ce qui peut affecter les marchés ", montre comment les informations hétérogènes sont mises en forme du point de vue de leurs répercussions économiques. Sous la notion de " faits économiques », on traite d'événements politiques, de nouvelles techniques de production et de communication, de matières premières, d'état financier de pays, de congrès internationaux industriels et commerciaux, de statistiques commerciales et industrielles en les rapportant à des logiques du marché. Ce quil y a de commun dans la facture de ces brefs articles est de nous montrer le caractère agissant du développement économique, alliage de l'essor de nouvelles techniques, de croissance des échanges internationaux, et son incidence sur le développement commercial et industriel du Canada. On y trouve également des informations sur une multitude de pays reliés aux empires coloniaux, on prétend mettre au jour une vision de la production et de la circulation économiques mondiales. Les rubriques de la section sont ordonnées de l'international au local, montrant bien par cette préséance du fait économique « universel » l'optique que cherche à promouvoir le directeur de l'école et de la revue.

La section « Revue bibliographique » est aussi diversifiée, alignant aussi bien des livres théoriques d'économie politique que des manuels techniques principalement reliés à la pratique des affaires en français, en anglais et en allemand. On trouve un ensemble de livres consacrés à des pays étrangers et au bilan de leurs activités économiques. La bibliothèque des HEC recevait des livres des ambassades, car l'enseignement aux HEC visait aussi à former des agents canadiens de commerce extérieur. Le contenu de cette section montre que ce travail de résumé-synthèse correspond au travail de recherche des professeurs qui en viendront à publier dans la revue des études sur des questions économiques sous forme d'articles plus développés.

Les articles hors section sont d'une ampleur beaucoup plus importante que les rubriques. On peut y constater la présence de collaborateurs universitaires étrangers (Georges Blondel, Léonard T. Reid, G. De Leener, F. Perroux, etc.), celle de professeurs des HEC (G. Le- 
chien, É. Montpetit, F. E. Fontaine, W. H. Athinton, P. Mercier, etc.) ainsi que celle d'experts du monde des affaires et de l'industrie. Une analyse a déjà été réalisée des affiliations géographiques des collaborateurs montrant l'importance des articles provenant d'auteurs français et belges. Un effort du tandem Laurey, directeur de la revue, et Minville, secrétaire à partir des années 1929, sera fait pour développer les collaborations européennes et donnera des résultats. Entre 40 et 50\% de collaborateurs d'Europe seront les auteurs des numéros (Dupré, Gagnon, Lanoie et CIRANO, 2000) dans les années 1930 27, montrant l'ouverture international de la revue.

Nous nous intéresserons particulièrement aux articles écrits par A.-J. Debray et É. Montpetit qui nous permettront de montrer comment est présenté l'économique comme fait humain universel. Encore plus que présenté, défendu, car plus sera connu ce travail, plus il suscitera de vives réactions dans le milieu clérical et politique jusqu'au déclenchement d'« une guerre de religion » menée contre les HEC (Harvey, 1994, p. 147).

On peut percevoir cette précarité de l'espace institutionnel dans un article publié par le directeur et intitulé « Pourquoi des Hautes Études Commerciales?» :

Voilà une question qui peut sembler quelque peu superflue. Et cependant, si on lit tout ce qui s'écrit sur l'enseignement commercial supérieur, si on écoute tout ce qui se dit, si on interroge même ceux qui devraient être renseignés de par leur situation, on constate, non sans surprise, que généralement l'on ne se rend pas compte de ce que sont les Écoles supérieures de commerce, du but qu'elles poursuivent, du mode d'organisation qui leur convient pour atteindre ce but. (HEC-REC, 3, 10, p. 334)

27 Plusieurs chercheurs attribuent à Minville le travail d'édition de la revue. Le dépouillement de la correspondance de la revue indique effectivement une séparation entre les fonctions de représentation assumées par Laurey et d'édition par Minville, qui rédige les lettres d'acceptation et les demandes de reformulation des articles auprès des auteurs européens comme locaux (Fonds des HEC, A0007). Si nous avons régulièrement la correspondance de Laurey, malheureusement celle de Minville est très parcellaire et ne peut nous donner à voir sa lecture et sa contribution à la mise en forme du discours de la revue. 
Le directeur des HEC poursuit ce texte en donnant l'exemple des grandes écoles commerciales des nations européennes et en faisant le bilan de leurs contributions au développement de leur économie respective. Les réactions furent fortes à la présence des HEC dans la société québécoise, comme le montre cette défense très polémique du professeur Debray dans la suite du texte :

L'histoire est là pour le montrer, que les périodes de grande activité économique furent les périodes les plus fécondes en savants de toutes sortes. Elle montre encore que les peuples qui dont pas su être suffisamment utilitaires, ont toujours été des peuples à mi-civilisation, qui ont fini par disparaître de la carte du monde [...]. Le jour, peu éloigné espérons-le, où la chose sera comprise au Canada, il ne sera plus nécessaire de répondre à la question pourquoi des Hautes Études Commerciales ? (HEC-REC, 3, 10, pp. 340-341)

L'arrivée d'économistes belges et français avec l'ouverture des HEC n'aura pas facilité la légitimation de cette école et celle de l'importance de l'économie, tout comme la reconnaissance des nécessités d'un utilitarisme. Cette menace à peine voilée du directeur de l'école, que la société québécoise demeure à mi-civilisation, nous permet de saisir où se situait la confrontation entre cette connaissance et l'univers idéologique d'alors : dans l'absence de nécessité de cette connaissance de l'économie, au fond, dans l'absence d'un problème économique identifié propre à la société québécoise d'alors qu'elle aurait à investiguer et à expliquer.

Au printemps 1913, le Livre des minutes des HEC fait état d'une altercation entre le directeur et le bureau de direction des HEC à propos des programmes d'enseignement qui seraient trop « théoriques »; on peut penser que cela signifie trop axés sur l'économie politique et la méthode « scientifique » des statistiques plutôt que sur les connaissances nécessaires aux affaires. Au mois de décembre, c'est la publication de la revue qui est remise en question par le bureau pour des raisons de budget déficitaire. En 1915, à la suite d'un rapport d'un membre du bureau au premier ministre, A.-J. Debray sera congédié. Les relations du directeur avec une société à « mi-civilisation »qui n'admet pas sa vision économique contribuent sûrement à cette affaire ${ }^{28}$. Hen-

Voir la note 25. 
ry Laury, économiste européen, déjà engagé comme professeur, le remplacera à la direction et continuera sous un mode plus diplomatique les mêmes objectifs de développement de l'école.

\section{3-3 Édouard Montpetit, médiateur}

\section{$\underline{\text { Retour à la table des matières }}$}

J'avais écrit dans un article de revue : «On pose le problème économique ; on le pose comme un blasphème, mais on le pose tout de même. » Transposition de vocabulaire plus ou moins heureuse. Naturellement, le mot sauta à la correction. je ne le regrette pas, mais ce trait traduit l'état d'esprit de cette époque : l'aspect matériel de notre problème national paraissait accessoire. (Montpetit, 1942, p. 19-20)

L'œuvre de Montpetit se résumera à faire admettre le problème économique, comme en fait foi la phrase leitmotiv de ses écrits : le problème national est un problème économique. Cette conception de l'économie de Montpetit trouve son fondement dans son approche du mouvement de l'histoire qui implique un rejet du déterminisme divin absolu : « [...] en premier lieu, le changement est un processus contingent et non déterminé sur lequel l'homme peut agir ; deuxièmement, la clef de cette contingence repose sur le jeu de deux influences, celle du milieu physique et celle de la culture, qui ensemble façonnent la société » (Southam, 197 1, p. 320). Il s'agit d'une conception qui, au lieu de percevoir l'homme comme soumis au divin ou aux forces de la nature, l'envisage comme actif, exploitant la nature, agissant sur sa propre réalité. Du même coup, cette définition de la société, se distanciant un peu plus du religieux, permet l'émergence d'arguments profanes qui ont pour objet l'économie et la société.

En 1921, dans un texte de L'Action française intitulé « L'indépendance économique des Canadiens français », Montpetit note une progression des mentalités attribuée non pas à l'effet des discours économiques, mais à la Première Guerre mondiale : « Il y a progrès. On reconnaît plus généralement aujourd'hui que la question économique importe. [...] L'événement a plus fait que les phrases alignées dans des revues sévères par des spécialistes, habitués aux horizons sans écho » (Montpetit, 1921b, p. 4). 
Cet article constituera la synthèse de l'enseignement d'Édouard Montpetit en économie politique, diffusé sous la forme de notes de cours la même année aux HEC. Ces documents nous permettent d'analyser différents aspects de sa pensée. Montpetit y affirme la présence d'un déterminisme économique universel qui transforme les Canadiens français :

Les besoins humains sont à la base de l'économie : plus ils se développent, plus la complexité de la vie économique s'accentue. Nous subirons la loi de l'extensibilité des besoins, n'étant pas autrement faits que le reste des hommes. Nous la subissons déjà : nos villes attirent chaque jour davantage l'homme que la terre ne satisfait plus, et ce qu'on est convenu d'appeler le « centre urbain » se modifie à vue d'oeil dans un sens qui n'est pas toujours heureux. La misère monte à côté de la fortune. Richesse ou dénuement, il peut arriver que l'on ait àchoisir. Nous touchons au problème individuel, dont l'angoisse est souvent la source des pires faiblesses. Les forces agissent. Il est temps que l'on accepte le mouvement économique pour le diriger en respectant les principes sains de la production. Ce qui ne se fera pas avec nous se fera sûrement sans nous et, peut-être, contre nous. (Montpetit, 1969, pp. 36-37)

En fait, la conception de l'économie de Montpetit a grandement fluctué au cours de sa vie. Elle est plus radicalement économique à ses débuts. Du point de vue théorique, les définitions données de l'économie sont pauvres, elles renvoient à la définition classique des mercantilistes : "L'économie politique a pour objet l'ensemble des activités humaines qui suscitent et distribuent les richesses. Ce dernier mot, dont vient tout le mal, fut ramassé par les fondateurs dans le langage populaire qui lui donnèrent un sens nouveau. [...] » (Montpetit, 1921b, p. 33). Dans ses premières publications, Montpetit définit l'économie à partir des caractéristiques communes aux économies développées en France, en Angleterre et aux États-Unis. Plutôt que de s'en tenir à créer des économies nationales fondées sur l'agriculture, les pays développés ont pu, une fois assurées leurs richesses, grâce à la création de manufactures royales et des premières politiques industrielles, hausser leur degré de civilisation vers un niveau de plus en plus élevé et, ainsi, se consacrer davantage aux tâches plus nobles de l'esprit. L'économie n'est donc plus la déchéance de la société chez Montpetit, mais bien plutôt un mal nécessaire pour pouvoir se consacrer à la vie 
de l'esprit. C'est pourquoi, dès ses premières prises de position publiques, Montpetit sera en faveur d'une industrialisation du Québec. Récemment revenu de ses années de formation européenne, il se fait radical pour l'époque en prônant le développement prioritaire d'un secteur secondaire orienté vers la substitution des importations ${ }^{29}$, comme l'affirmeront beaucoup plus tard les économistes de la dépendance. Cette définition du problème national comme un problème économique a des conséquences au-delà de l'économie.

La première définition de l'économie québécoise qui ressort de ces travaux renoue donc celle-ci à la nature, comme le mentionne Édouard Montpetit dans sa critique des écrits de Bouchette : «... Bouchette [...] dit que le capital national est l'ensemble des ressources exportables d'un pays. Il s'appuie sur Robertus Jaketzov (Davenport également). C'est confondre la nature et le capital. Mais cette théorie est ingénieuse et féconde. Elle nous fait croire en notre force » (Montpetit, $1921 \mathrm{ab}$, p. 50).

En fait, Montpetit lui-même ne se démarquera pas vraiment de cette conception dans ses descriptions de l'économie québécoise. Cette conception de l'économie comme nature se définit méthodologiquement aussi par l'approche monographique qui a comme caractéristique générale un "souci de totalisation ». La monographie fut pratiquée pour mettre en relation le milieu physique et celui de la culture, tel que le proposait Montpetit. Cependant, la monographie n'est pas la seule méthode utilisée. Le premier directeur des HEC, A.-J. Debray, est un économiste européen spécialisé en statistiques qui participe du

29 « De l'étude comparée des chiffres de notre commerce extérieur, M. De Bray tire une conclusion : "Les trois quarts de nos importations sont des produits manufacturés et les trois quarts de nos exportations sont des produits naturels. » Notre attention doit être retenue par les avantages qu'il y aurait à"transformer nous-mêmes, notamment en assurant à ceux-ci un débouché" C'est poser toute la question de notre avenir économique et montrer toute la nécessité d'une organisation de nos forces : nous sommes tributaires de l'étranger; nous lui vendons les matières premières dont il a besoin et nous lui abandonnons ainsi les bénéfices réels auxquels nous avons droit » (Montpetit, É. [1914], « L'essor industriel et commercial du peuple canadien », Revue économique canadienne, 3, 7, pp. 231-237). 
développement de cette méthode à l'échelle internationale ${ }^{30}$. Il publiera un bilan de l'économie canadienne en 1914 sous le titre L'essor industriel et commercial du peuple canadien, ouvrage principalement écrit à partir de statistiques fédérales à l'échelle du Canada. Montpetit se faisant médiateur publiera sous le même titre un compte rendu de cet ouvrage sous la forme d'un vif plaidoyer en faveur de l'approche statistique. Face à un public habitué aux monographies économiques, celui-ci défendra la pertinence de cette réduction du réel qu'opèrent les statistiques :

On nous pardonnera cette apologie de la statistique, vraiment, les chiffres qu'elle aligne paraissent parfois nous inspirer une sorte de crainte respectueuse ou d'horreur instinctive. Et pourtant, ils sont bien le signe de la vie. Comment connaître sans eux, la valeur exacte d'une richesse ; comment supputer le nombre et l'efficacité d'une force ; comment jalonner les étapes d'un progrès et marquer la marche d'une civilisation ? (HEC-REC, 1914, p. 121)

Ce premier débat dans une science sociale au Québec, entre une approche qualitative et une autre quantitative, met en valeur l'irréductibilité du fait humain à l'un de ses aspects, réduction qui se ferait sous peine de perdre son essence, voire son âme, pour reprendre les termes de l'époque ${ }^{31}$.

Dans cette mise en évidence des relations de plus en plus étroites entre la société québécoise et l'économie environnante, la perspective

30 En 1913, Debray est devenu membre de la Commission internationale pour l'enseignement de la statistique dans les universités (Harvey, 1994, p. 239).

31 De plus, le portrait descriptif livré par les statistiques économiques fédérales ne donnait lieu qu'à des déductions très approximatives à propos de l'économie francophone au Québec. Ce sera le cas jusqu'aux années 1960 où le gouvernement du Québec créera un office de la statistique qui inclura des distinctions de propriétés selon l'origine ethnique. L'économiste André Raynauld fut le premier à réaliser dans les années 1960 un sondage selon la méthode scientifique où il inclura ce qu'il appelle des variables culturelles, notant que cellesci n'ont aucun statut du point de vue de la théorie économique bien qu'elles soient celles qui déterminent le plus les variations des données économiques au Québec. Voir Raynauld, A. (1974), La propriété des entreprises au Québec les années 60, Montréal, Presses de l'Université de Montréal, p. 10. 
de Montpetit sera déchirée par le problème d'un développement capitaliste de la société québécoise : " nous pouvions être capitalistes : en fait, nous l'étions » (Montpetit, 1969, p. 32). C'est ainsi que le travail de Montpetit, plus radical à ses débuts, ayant comme modèle les pays développés, donnera lieu à un discours fabriqué de statistiques et de citations d'économistes établis, sans toutefois que ne soit exposée, à proprement parler, une théorie de l'économie politique. Or, plus Montpetit tente de diffuser le discours économique, plus son discours s'édulcore en un discours moral, voire religieux, allant jusqu'à énoncer son programme d'action comme une doctrine. Les responsabilités administratives qu'il cumule au cours des années 1920 et qui se multiplient par la suite contribuent sûrement au peu d'actualisation de sa connaissance de l'économie politique ${ }^{32}$. Entre ces deux moments, on assiste au Passage d'une définition de l'économie comme problème national à l'analyse d'un aspect économique du problème national qui, lui, est défini dans l'univers religieux. La relation entre la société québécoise et son économie peut être conceptualisée comme une problématique contradictoire. Entre le constat que Montpetit fait en écrivant que "L'intérêt individuel guide, dans la réalité, le monde économique », et celui voulant que " La richesse, nous l'avons dit, n'est qu'un moyen : la satisfaction morale dans la paix respectueuse et le bien-être assuré est le but suprême »(Montpetit, 1969, p. 36), il y $a$ un fossé qui sera comblé tant bien que mal par les ressources stylistiques de l'essai.

L'essai, qui sera la stratégie d'écriture de ces penseurs de il économie, a comme intentionnalité la persuasion. Cette mise en forme est à même de conférer une cohérence à des arguments contradictoires, mais ce n'est pas grâce à un procédé entièrement déductif ni dans le tourbillon du chassé-croisé des nombreux arguments qui succèdent aux prémisses, mais surtout à sa capacité à faire percevoir ses incohérences comme étant irréductibles à la perception du réel communément admise. La connaissance immanente se voit conférer le statut de l'illusoire. Ceci différencie ce discours d'une connaissance scientifique qui pose un ordre du réel nécessaire à découvrir et postule qu'il est

32 En 1920, il fonde l'École des sciences sociales économiques et politiques de l'Université de Montréal et est nommé secrétaire général de cette université. Il assume plusieurs tâches d'administration ainsi que plusieurs autres missions pour le gouvernement du Canada et celui du Québec. 
possible d'en produire une représentation mettant en jeu l'observation du monde. Cette incohérence du réel avec les lois divines, relatée par Montpetit, nous la retrouvons dans cette citation à propos de la construction d'un édifice à des fins sociales :

La construction d'une université ou d'un hôpital est une question d'argent. L'oeuvre d'instruction et l'oeuvre d'assistance s'érigent sur une première pierre, solide et durable, triste matière. Certes, le sacrifice pourrait faire beaucoup, entasser les matériaux, dresser les murs, déployer les toits, lancer les flèches ; mais nous parlons pour notre siècle et notre moment. Le mal est fait. (Montpetit, 1969, pp. 33-34)

Ce mal, on l'aura deviné, c'est l'économisation de la société canadienne-française : le passage d'une société où les édifices publics étaient bâtis dans une économie «du sacrifice » à une société où ils seront désormais bâtis «par l'argent ». D'où les enjeux de cette problématique : si les lois divines transcendent le réel, quel est ce nouveau réel qui ne correspond pas aux anciennes façons de faire et de penser, qui semble contraire aux règles religieuses puisqu'il apparaît comme un mal ? En fait, la question de la compatibilité entre la doctrine catholique et la science économique de même que celle d'un comportement économique moral face au comportement économique capitaliste rentable seront résolues par un effet de style plutôt que par la prise en compte de l'incompatibilité de ces connaissances contradictoires. Nous pouvons résumer cette résolution ainsi : la science économique devra être un discours moral et démontre en quoi l'économie est une réalité tout aussi morale.

La moralité est un élément constituant de la science économique. En veut-on un exemple arrêté au hasard ? Sur quoi repose la production, l'ordre, que demande-t-on aux pays producteurs ? Des hommes, et le principe de population est radicalement un principe moral. Qu'est-ce que l'homme même moteur initial des activités économiques ? Un être raisonnable. Que réclame-t-on de l'ouvrier, outre l'habilité, la conscience ? (Montpetit, 1969, p. 35)

La science économique d'alors reste un discours défini par le religieux même dans les notions propres à ce domaine de connaissance. Si elle emploie les statistiques qui donnent une vision fractionnée de la réalité humaine, c'est en conciliant cette approche avec la méthode 
monographique plus respectueuse de son intégralité. Ce n'est pas uniquement la science économique qui est Morale, mais, malgré toutes les apparences, l'économie en soi. En écrivant en 1931 son article sur « La pratique et la doctrine sociale catholique », Montpetit compare le discours papal à celui d'un homme d'affaires (Rockefeller) et constate que « les magnats d'industrie ont des affirmations qui, sans les confondre, les unissent dans une même préoccupation d'humanité " (Montpetit, 1969, p. 37). Ces affirmations, qui nous semblent naïves sachant aujourd'hui la source de ces grandes fortunes américaines, ne peuvent s'expliquer que par sa façon d'appréhender l'économie, perçue comme venant de l'extérieur, comme un simple moyen à utiliser avec modération, « un moyen vers l'expansion, la totalité, le social ».

Ces affirmations renvoient à l'ensemble des contradictions caractéristiques de la pensée économique de cette époque ; contradiction entre les constatations empiriques de l'impact du capitalisme entrevu comme un profond bouleversement et la réduction de l'économie capitaliste à un simple moyen technique, contradiction à la volonté de fonder une démarche réductionniste, une science de l'économie, tout en conservant une approche de la totalité du fait humain. La résolution de ces contradictions se fera d'elle-même dans un avenir projeté, car les lois divines sont les lois de toutes les activités humaines et donc, on comprendra un jour la manifestation des principes chrétiens dans l'activité économique. De toute façon, dans un avenir à moyen terme, le développement de l'économie permettra à la société de venir (ou de revenir ?) aux choses de l'esprit, à une vie spirituelle. La pensée médiatrice de Montpetit se posera à terme dans une conception du savoir doctrinal comme un prolongement du rapport de connaissance religieux qui marque l'univers idéologique de son époque. Par ailleurs, malgré cela, Montpetit ne cessera pas d'appuyer par ces gestes le développement de la connaissance économique que va entreprendre la seconde génération d'économistes québécois et qui lui conférera une relative autonomie.

À la différence de Montpetit, on peut observer dans le travail d'Esdras Minville une prise en compte, au moins partielle, des médiations de la connaissance de l'économie lorsqu'il critique et tente de réorganiser le savoir d'économie politique dans une nouvelle conception de la connaissance : une science économique appliquée à même de cons- 
truire une lecture d'une économie francophone qui se laisse difficilement décrire avec les catégories et les méthodes de la science économique, comme l'économiste Raynauld (1974) le montrera plus tard.

\section{L'actualité économique: le travail de déconstruction de l'économique comme universalisme}

$\underline{\text { Retour à la table des matières }}$

Onze ans après l'abandon de la publication de la Revue economique canadienne, le projet est repris par un groupe de nouveaux diplômés, dont les plus connus sont Gérard Parizeau, François Vézina, Jean Nolin et Esdras Minville. Le discours économique se heurte encore aux résistances de l'opinion publique. Dès le troisième paragraphe de sa présentation, le directeur de l'école, Henri Laureys, définit la mission de la revue : « ... elle prouvera aux pessimistes à courte vue que, malgré leurs vaines et puériles attaques, ceux qui depuis quelques années se sont résolument tournés vers les professions commerciales et industrielles, vraiment créatrices de richesses, sont dans le vrai... » (Laureys, 1925, p. 1). De plus, dans une nouvelle section « Tribune libre », une lettre ouverte signée sous un pseudonyme défend ardemment la formation académique des HEC.

Aux rubriques de l'ancienne revue s'ajoutent une section sur l'information financière puis, à partir du troisième numéro, une chronique sur la bourse de Lucien Favreau. Cet accent sur la dimension financière de l'activité économique est donc nouvelle et correspond à un travail d'analyse du capitalisme qui mènera à la mise en cause de l'universalisme économique comme acquis de civilisation au point de le dénoncer comme un impérialisme économique.

Esdras Minville, dans trois articles qui prennent la forme d'essais ( "Les Américains et nous », « Le capital étranger » et « Agir pour vivre »), pourfend l'impérialisme américain qu'il conçoit comme un nouveau stade de l'impérialisme colonial d'origine européenne. Le 
monde économique ne se résume plus au progrès par le développement économique, mais est constitué de rapports de domination coloniale qui s'élaborent maintenant à travers l'économique à l'échelle de nombreux pays ${ }^{33}$. L'espace social de la pensée économique d'Esdras Minville n'est pas local ou national, mais bien international ${ }^{34}$. Il se situe en filiation directe avec Édouard Montpetit, son professeur, qui a clamé l'indépendance économique du Canada français. Cependant, le type de travail intellectuel qu'il va accomplir sera différent de celui de Montpetit. Contrairement aux autres professeurs des HEC qui vont entreprendre des études supérieures en Europe, Esdras Minville travaillera après ses études de premier cycle dans une maison de publicité. Plutôt que de se marginaliser du monde académique, celui-ci assumera, après être devenu professeur de rédaction publicitaire aux HEC, la direction de L'Actualité économique puis celle de l'école de 1938 à 1962. Sa connaissance plus approfondie de l'économie résulte d'un travail acharné de synthèse de l'information économique nationale et internationale, mais aussi d'une ouverture exceptionnelle aux autres disciplines des sciences sociales, ouverture qui sera acquise par un travail autodidacte lui évitant ainsi la conformité du « moule » que constituait l'économie politique classique. Ce travail d'autoformation,

33 C'est du fait que Minville pose l'économie dans ses rapports de domination impérialiste que l'on peut comprendre le rôle de précurseur des sciences économiques du XXe siècle que lui accordait l'économiste français François Perroux, élève de Schumpeter, qui a réservé lui aussi une place majeure à cette question de la domination dans son oeuvre. Plusieurs parallèles pourraient être faits entre leurs œuvres, dont ceux de saisir les limites du modèle keynésien, de proposer un dialogue entre théorie économique et histoire, de privilégier les formes collectives d'organisation de l'économie, etc. François Perroux publiera trois de ses articles dans la revue L'Actualité économique. En 1959, il ira jusqu'à suggérer dans une lettre à Esdras Minville, alors directeur des HEC, de modifier le statut du Centre de documentation économique des HEC pour celui d'Institut pour permettre son affiliation à l'Institut de Paris dirigé par François Perroux. Ce qui sera fait (Charbonneau, 1991, pp. 3-4).

34 Ce sera le cas aussi de plusieurs sociologues de l'économie marxiste à cette époque (Arnaud Sales, Jorge Niosi) et d'économistes, tel Jacques Parizeau, qui, dans les années 1960, poseront d'emblée que la bourgeoisie a des comportements nationaux. Le phénomène de la mondialisation économique montre qu'on ne peut présumer cette configuration sociale des comportements économiques. 
Minville le réalisera en se donnant des programmes de lecture, à travers la rédaction des sections "Faits et nouvelles », ses contributions à la revue des périodiques et des livres de L'Actualité économique, et ce travail se poursuivra pendant plusieurs années : à partir de 1928, il assumera la direction de cette revue.

4-1 Le travail intellectuel de la « science " économique appliquée ou la genèse de la connaissance administrative

$\underline{\text { Retour à la table des matières }}$

"L'homme est une synthèse », écrit Minville ; les médiations de son travail, il en fait la métaphore de sa conception de l'être humain. La raison doit expliciter et actualiser cette synthèse. Le terme de synthèse suppose que la matière du vécu se présente comme des fragments d'existence comme ceux des « Faits et nouvelles », mais aussi, que les interprétations que sont les idéologies économiques se déclinent comme des thèses, des antithèses qui appellent l'activité de synthèse. Voilà résumé brièvement le statut que Minville accorde au travail de connaissance opéré par cette " science » économique appliquée et qu'il explicite graduellement jusqu'à écrire un livre sur cette question, l'Invitation à l'étude. L'examen des "Faits et nouvelles» nous montre une lecture conférant des statuts différents aux lois économiques, aux faits économiques, aux statistiques économiques et à la doctrine économique. L'ampleur du travail intellectuel accompli et son caractère très diversifié à partir des journaux généraux et spécialisés, des revues économiques européennes et américaines, des documents financiers publics et privés rendent compte de sa lecture originale de l'économie. Comment sa quête de raison peut-elle démêler l'écheveau de cette hétérogénéité des discours?

Le discours public, peut-on lire dans un de ses articles, est tenu par les « discoureurs » et journalistes qui apparaissent comme « un monceau de glose, de harangues, de discours, d'articles de revues et de journaux de tout format et de toute nuance... [qui] s'appuyant sur les mêmes données statistiques... trouvent moyen d'édifier des démonstrations également probantes et pourtant des conclusions diamétralement opposées » (AE, 1925, p. 12). Selon Minville, ce caractère inextrica- 
ble de l'argumentation économique dans les journaux relève de l'usage politique des statistiques : « le prisme déformant d'une étroite partisanerie ». Est-ce que le domaine du discours savant sur l'économie permet de trancher entre les diverses argumentations ? La réponse que l'on peut lire dans les travaux de Minville est variable, et montre comment il se déplace entre différents points de vue sur l'économie politique. Dans les « Faits et nouvelles », il pose qu'il y aurait existence de lois économiques dont la valeur peut être tranchée par leur confrontation aux statistiques économiques. Dans un écrit sur le chômage, par exemple, il confronte les thèses de l'économiste français Jacques Rueff, qu'il tire de la Revue d'économie politique, avec celles de deux autres économistes français qui les réfutent et dont les articles ont été publiés dans la Revue internationale du travail et la Revue d'économie politique. Ce qui va permettre à Minville de trancher entre les différentes approches est la confrontation avec les statistiques allemandes dans l'étude de $\mathrm{M}$. E. Thiele qui le mènera à la conclusion que « la prétendue loi découverte par celui-ci à l'aide des statistiques britanniques ne se vérifie pas en Allemagne, il n'en est donc rien, puisque cette loi économique, si elle existait, devrait s'appliquer à tous les pays » (AE, 1932, 5, 3, p. 69). On reconnait ici le principe de généralité mis en cause par la falsification que permet un cas observé. Mais le statut de faits proprement économiques reconnus est ambigu comme l'indique le point d'interrogation dans l'article «Plus de respect des lois économiques ? " qu'il écrit dans le cadre des " solutions » à apporter à la crise des années 1930 : « l'activité économique obéit à un certain nombre de lois naturelles dont les événements ne cessent de confirmer l'exactitude ». Il reprend ici le plaidoyer de l'économiste français André Liesse pour dire que si des praticiens ont ignoré ces lois, il s'ensuit la « sanction inéluctable des lois économiques ». Cependant, quelques lignes plus bas, diagnostiquant la crise des années 1930 comme une crise de la répartition, il va s'opposer à la dénonciation par Liesse de l'intervention sous toutes ses formes dans le « jeu des lois économiques ». Reprenant la valeur de liberté du libéralisme économique, il la met en relation avec celle de justice : " Il n'y a pas de véritable liberté que dans la justice. » Si les lois de l'économique sont des lois naturelles (du monde physique et de la part physique de l'humain) qui traduisent une réalité économique, ces lois ne sont pas « intangibles », c'est-à-dire hors de la portée de la raison et de l'intervention. Il y a donc une autonomie du champ de l'économique, 
mais cette autonomie est limitée par un appel à des valeurs extérieures au champ. Cette vision restrictive de l'économique est aussi due à sa méthode privilégiée, les statistiques. Minville se range à la vision de Montpetit, qui fait de la statistique une méthode scientifique non sans montrer son hésitation en recourant à une élision bien québécoise : « Mettons-le 35 aussi »! (AE, 1933, 6, 2, p. 27). En fait, il perçoit très bien la construction de l'information à travers les statistiques, ce qui est plutôt rare chez les économistes de l'époque. La mesure du PIB implique des opérations d'interprétation : "C'est ce que font les statisticiens, en se livrant à des appréciations et à des interpolations qui aboutissent à des approximations qui, tout en ne prêtant nullement à une rigoureuse exactitude, sont néanmoins fort instructives »(AE, 1933, 6, 2, p. 30). Il y a donc un aspect normatif irréductible dans le travail du statisticien qu'explicite Minville dans ses interprétations statistiques. Toutes ces nuances s'évanouissent à certains moments, probablement sous la pression des échéances de publication ; Minville reconduit alors tout simplement les lois économiques sous un mode universalisant, telle la « loi » de la rationalisation du travail dans un compte rendu de la rubrique « À travers les revues » (AE, 1931, 6, 10, p. 376).

Minville a un rapport critique aux discours. Ce travail critique dans l'œuvre de Minville ne s'applique pas uniquement à l'économique doctrinaire, il s'applique aussi au religieux doctrinaire qui ne distingue pas entre l'esprit religieux et ses manifestations extérieures :

Ainsi en est-il de la pratique religieuse et de la religion. Bien qu'en très intime relation, la première n'est que la manifestation extérieure de la seconde... Le R. Père Doncoeur, s.j. n'hésite pas à présenter comme grand catholique, - voire - ce qui implique peut-être davantage - comme grand écrivain catholique, Charles Péguy qui, sur la voie du retour n'était pas parvenu à la pratique religieuse. Mais s'il est juste qu'à un certain point de classer comme bons catholiques des non-pratiquants, en revanche, il n'y a aucun risque à classer certains grands pratiquants comme de tristes catholiques. (Minville, 1943, pp. 73-74)

Si des travaux ont traité en termes d'influences de la pensée économique (l'école du nationalisme économique) et de la philosophie

35 « Mettons-le » doit être compris comme « admettons-le ». 
sociale de Minville (L'Action française et l'abbé GROULX) ${ }^{36}$, il faut aussi faire état de sa connaissance de la vie et de l'œuvre de Charles Péguy qui est essentielle à la compréhension de ses travaux. Plus que des idées sur l'importance du nationalisme, du socialisme, Minville absorbe et reconstruit une connaissance qui implique une distanciation

36 Pour une description thématique de la pensée économique de L'Action française, voir Dupuis (1992, chapitre 1). Pour résumer trop brièvement notre position dans le cadre de cet article, l'analyse en termes d'influence, qui a donné lieu aux débats relatifs à l'agriculturisme de ces penseurs et par extension à leurs résistances ou non à l'industrialisation, nous semble discuter sur des bases problématiques. Lorsque l'on appelle les travaux de William F. Ryan sur l'attitude du clergé face à l'industrialisation, il faut faire attention à ne pas projeter le sens du mot industrialisation d'aujourd'hui sur hier d'autant que ce terme définit un processus social en le résumant à son aspect technique. En termes sociologiques, l'économie industrielle est toujours composée de relations sociales. Avec des collègues, nous avons produit une sociographie des activités d'entreprises francophones durant cette période pour montrer que les relations de parenté et d'alliances, puis ces relations redéfinies en termes ethniques, composaient le processus d'accumulation qui produisait non pas une capitalisation des entreprises mais un processus de redistribution sociale menant vers la décapitalisation (Houle, Hamel et Sabourin, 1984). C'est ce genre d' " industrialisation », non sans réserves et contrôle de la part du clergé, que celui-ci a appuyé. La pensée économique de L'Action française s'est forgée à travers l'appropriation de l'économie politique de l'école du nationalisme économique (Dupuis, 1992, p. 83). Par ailleurs, la transposition de la vision de Groulx à Minville oublie que l'essentiel du travail effectué par Minville est de produire un savoir économique, non pas une sociologie pas plus qu'un travail historique tandis que Groulx ne s'intéresse pas à l'économique (Dupuis, 1992, p. 29). Il a bien des points communs à leurs pensées : la conscience nationale, mais Minville voit dans l'éducation, la recherche et les coopératives, plutôt que le politique, le heu des transformations de la conscience nationale. 
critique et un souci du présent ${ }^{37}$, qui l'oblige à reconstruire sa pensée à partir d'un travail constant d'observation de la pluralité des «faits actuels ». Il se situe donc à l'opposé de ce que dénonceront les durkheimiens en France, c'est-à-dire une science économique dont le travail d'observation est absent. Cette distance critique n'est pas toujours maintenue, particulièrement aux moments les plus graves de la crise des années 193038 .

Décrire ce travail de connaissance permet de mieux comprendre la lecture rétrospective de l'économiste québécois Pierre Fortin qui se questionne sur la rupture drastique qui aurait été induite par sa génération à la suite de la formation nord-américaine " scientifique » des économistes après la Seconde Guerre mondiale. Cette " rupture » pourrait avoir été en fait provoquée, " ce sont souvent les aînés, formés en Europe, qui ont orienté leurs meilleurs étudiants vers l'Amérique » (Fortin, 1984, pp. 167-168), jouant donc ainsi plutôt un rôle de médiateurs en facilitant l'accès à cette formation nord-américaine.

37 Françoise Gerboud résume le point de vue de Peguy de la façon suivante : «Selon lui, il faut maintenir dans le jeu des événements la fidélité à la source profonde de ses engagements qui oblige à revoir constamment ses analyses, à rebondir de façon neuve. Cette fidélité, il la nomme mystique. Or la mystique de Péguy est avant tout libertaire. Il revendique le droit à une pensée fibre de toutes les autorités, dénonce ce qui pourrait devenir "une république socialiste gouvernementale" Il refuse qu'une certaine conception de la laïcité fasse de l'athéisme "une métaphysique d'État" Sa mise en question des dérives autoritaires du socialisme, si elle peut paraître excessive au regard des événements qui lui sont contemporains, est pourtant prémonitoire. Il nous est plus facile d'en mesurer la validité en des années où l'idéologie communiste a fait faillite. Péguy défend le droit au pluralisme politique et philosophique. Toute grande philosophie est pour lui une voix essentielle de l'humanité. Il ne considère pas que les philosophies forment une suite linéaire et progressive ; elles restent diverses et continuent à dialoguer entre elles au cours des siècles » («Charles Peguy », Dictionnaire Universalis, 2004).

38 Par exemple, dans une conférence écrite en 1938 et publiée par les Semaines sociales du Canada ("Pour une société chrétienne »), il affirme le catholicisme comme un universalisme : « La doctrine sociale à laquelle nous adhérons et à laquelle le monde entier, sous peine de ruine, devrait se rallier tôt ou tard, c'est celle de l'Église catholique. Cette doctrine fonde les relations sociales sur la justice et la charité »(AE, octobre, 1938). 


\author{
4.2 La valeur d'usage \\ comme lieu de médiation conceptuelle \\ et le refus de l'autonomisation de l'économique
}

$\underline{\text { Retour à la table des matières }}$

Selon Minville, la discipline-synthèse par excellence est la géographie qui englobe déjà l'observation de la nature et de la culture. L'espace de médiation entre la nature et la culture réside dans la notion d'« usage ». Sa conception de la valeur d'usage est posée d'une façon originale dans son discours économique par son statut de forme construite : " C'est lui qui (l'homme), par ses idées justes ou fausses, par ses besoins réels ou fictifs, établit le premier lien avec le monde qu'il habite, avec les choses qui l'entourent. " La rencontre en 1926 lors d'une conférence aux HEC avec le géographe Jean Brunhes, professeur au Collège de France, sera déterminante de sa démarche de connaissance tant du point de vue « théorique »que méthodologique.

À l'universalisme de l'échange économique, Minville substituera la lecture générale de l'histoire humaine de la géographie de Brunhes qui répond à des « questions philosophiques fondamentales » pour celuici, montrant par ce commentaire de quel point de vue il assimile ce discours.

La houille n'existait virtuellement pas avant que l'homme conçoive l'idée de l'utiliser. Ayant reconnu les propriétés de cette espèce minérale, en décidant de l'exploiter, il pose un acte causal (au sens du plus déterminant)... L'homme se forge des besoins qui deviennent bientôt des nécessités. Il domine les choses, mais l'usage qu'il en fait le rend dépendant. Agissant lui-même sur le milieu, à ne peut en éviter l'emprunte et les conséquences de cette influence réciproque se reflètent dans la physionomie du monde. (AE, 1926, 2, 1, p. 11)

Autrement dit, l'activité de pensée dans le mouvement de la culture construit l'usage des objets et est déterminante du mouvement économique qui vient par la suite exercer des déterminations (au sens de 
contraintes) sur la vie humaine ${ }^{39}$. Ainsi Minville, plutôt que de constater l'inexorabilité de l'extension illimitée des besoins humains comme Montpetit, va tenter de retravailler la notion d'usage dont la géographie cumule les traces. Ainsi, la normativité est au centre de la constitution de l'économique comme "science ", brisant du même coup l'idée d'autonomisation de l'économique justifiée sur la base d'une valeur d'usage intrinsèque aux objets.

Mais comment reconstruire cette pensée humaine agissante qui fixe des besoins et génère des conceptions de futilité des êtres et des choses ? Peut-on dégager une raison culturelle qui fonderait l'élaboration d'autres utilités que celles existantes ? Est-ce que l'économie capitaliste est seulement un moyen que l'on peut soumettre à la création d'un usage conforme à la culture québécoise?

Les chemins qui vont s'ouvrir dans cette « brèche », chemins médiateurs de l'affrontement des savoirs économiques et des savoirs culturels, vont être multiples. Des chemins ordonnés d'Invitation à l'étude où, malgré la pluralité des valeurs, la projection interrogative du travail de connaissance laisse présager, dans une synthèse future de la recherche, un ordonnancement des valeurs à partir de celles issues de la transcendance religieuse, mais aussi des chemins plus chaotiques, ceux des constats incertains sur le capitalisme, du dédoublement de la réalité dans les catégories abstraites de l'économique et du social, du caractère transitoire plutôt qu'instituant des alternatives économiques (coopératisme, corporatisme) au moment où la crise du capitalisme des années 1930 rendait tout envisageable. Nous ne pourrons analyser tous ces chemins empruntés par Minville. Ce serait l'objet d'un article en soi. Concentrons-nous plutôt sur les tensions et leur aménagement pour constituer un " nouveau mode de connaissance de l'économie » adapté aux sociétés subissant l'impérialisme économique et qui, « du

39 Ce n'est que dans les perspectives hétérodoxes contemporaines, par exemple celle de Jacquemin (1985) définissant « la sélection et le pouvoir dans la nouvelle économie industrielle », que nous trouvons ce genre de réflexion sur la construction culturelle de l'usage en référence à la définition de la culture selon Claude Levi-Strauss. On y montre de plus que cette fabrication culturelle de l'usage est aux fondements des rapports économiques concurrentiels aujourd'hui au même titre que l'économie d'échelle. 
fait que la société canadienne-française est catholique », doit produire un mode d'intervention rationnel respectant les valeurs définissant cette société. Le savoir doctrinaire de l'économie politique ne constitue pas le bon chemin puisqu'il ne permet pas de trancher sur les méfaits du capitalisme que Minville dénonce par ailleurs en mettant en évidence la prolétarisation des Canadiens français :

Enfin, dernière remarque : le régime sous lequel nous vivons est libéral, mais aussi capitaliste. Et s'à y a désordre dans le monde, la question ne mérite-t-elle pas d'être posée de savoir quel est le principal coupable, du libéralisme ou du capitalisme, ou des deux à la fois, et dans quelle mesure ? Là-dessus, les opinions sont partagées à l'excès. Après des années de dispute, on est loin d'en être arrivé à l'ombre d'une entente. C'est que selon que l'on charge davantage ou le libéralisme ou le capitalisme, les conclusions diffèrent profondément pour ne pas dire se contredisent. (AE, 1936, 2)

Cette citation nous permet de comprendre pourquoi Minville s'est orienté vers le développement d'un savoir appliqué et des recherches empiriques sur l'économie québécoise, lesquelles s'inspirent d'une connaissance des expériences économiques de par le monde. Les travaux sur l'exploitation rationnelle de la forêt, qui anticipent les débats que nous connaissons aujourd'hui, en constituent un bel exemple : «Minville formule là ce qui deviendra le cœur de l'approche coopérative de la colonisation : l'exploitation de la forêt selon une logique patrimoniale, aux exigences radicalement opposées à celles de la logique de prédation mise en oeuvre par les grandes compagnies forestières avec le soutien et la complicité de l'État » (Laplante, 1994, p. 70). Néanmoins, nous retrouvons chez Minville, à d'autres moments, des prises de position « théoriques » qui tranchent, où le capitalisme n'est qu'un moyen technique de rationalisation de la production qui peut être réorienté pour assurer les fondements sociaux de la société canadienne-française : "L L'économique, au contraire (du social) n'a pas de relation directe avec la culture et les autres valeurs nationales » (Minville, 1979, p. 200). Si la totalisation qu'il induit par la description de la société canadienne-française est faite par l'identification d'une pluralité de valeurs (religieuses, culturelles, sociales) où le religieux prédomine dans l'ordonnancement, par ailleurs chacun des domaines a une autonomie relative et ils exercent entre eux des influences réciproques. Seul l'économique comme technique de production, et non 
comme valeur, n'exerce pas directement de détermination sur les autres au sens où une société choisit son économie et non l'inverse. Et pourtant, cherchant à asseoir l'objectivité de cette notion de société afin de créer des projets économiques compatibles, Minville fait d'autres constats que ceux-ci, en empruntant l'analyse des cadres sociaux au sociologue Léon Gérin :

Pour comprendre sa situation, le drame de son existence, il ne suffit pas de décrire ses mœurs et ses modes de vie à un moment donné ; il faut la situer dans sa perspective historique, dans la ligne de son passé, traversé de tant d'épreuves et de si diverses. De cadres sociaux, quand le vieux régime familial a été ébranlé, puis dispersé, eue n'en a trouvé que le simulacre, la caricature : les partis politiques. Elle s'y est embradée, comme d'instinct, à la recherche d'une sorte de sécurité. Elle n'en sortira pas - quelles que représentations qu'on en fasse - tant que d'autres cadres, vraiment sociaux ceux-là, n'auront pas été dressés pour la recevoir. Nul mieux que Léon Gérin n'a décrit (sans l'expliquer cependant) la tragédie de la famille canadienne-française d'ici il y a un demi-siècle, tiraillée, écartelée même par des influences dont elle ne déchiffrait pas l'énigme et qui persistaient, par une sorte de mouvement spontané, de réflexe héréditaire, à se reconstituer selon une formule dépassée, dans un cadre qui déjà de toute part cédait, s'évanouissait. (AE, 1938, p. 344)

De la même façon que les valeurs doivent être réactualisées par la raison dans l'appréhension des faits nouveaux, les cadres sociaux de la nation canadienne-française ne se reproduisent plus : une politique économique s'impose dès lors pour les réactualiser. 


\section{Conclusion}

Retour à la table des matières

En conclusion, du point de vue d'une sociologie de la connaissance, l'enjeu de cet article n'était pas seulement d'analyser le fondement normatif de la pensée économique explicitement catholique. Il s'agissait aussi de comprendre comment, malgré ce fondement normatif, cette seconde génération des penseurs de l'économie est parvenue à saisir lucidement que cette transition de l'économie et de la société québécoise sapait les cadres sociaux traditionnels auxquels ils adhéraient fortement de par leur socialisation (Houle, 1983), dépassant ainsi une conception locale ou universelle du savoir économique. Autrement dit, nous avons tenté de montrer que dans l'œuvre de Minville apparaît, dans ses termes, la localisation sociale de l'économie canadienne-française, mais aussi de sa connaissance par le rapport critique qu'il arrive à développer. À partir des années 1950, Esdras Minville posera la problématique de la relativité qui caractérise la forme de connaissance qu'il a tenté de constituer à la mesure d'une époque et d'une société :

Le problème ainsi posé est d'ordre métaphysique, voire théologique : il s'agit de savoir comment les hommes de la génération présente et des générations à venir interpréteront en eux-mêmes et dans leurs relations réciproques, les éléments de la réalité intégrale : nature, personne humaine, société, histoire. En dernière analyse, il s'agit de savoir selon quelle conception de leur vocation d'homme ils organiseront leur vie. Mais on ne pose pas le problème de l'homme sans interroger aussitôt ses rapports avec Dieu. (Minville, 1979, p. 57)

Nous avons cherché à montrer que les médiations sociales dans le travail de Minville ne résultent pas en une synthèse, mais produisent plutôt un mouvement d'objectivation de la localisation sociale de sa connaissance qui « interroge » ses fondements jusqu'à ceux de son propre rapport au monde. Ce statut explicite de la normativité, qu'il confère dans son travail de connaissance, nous semble expliquer le 
fait qu'Esdras Minville, au moment de sa direction des HEC, a plutôt favorisé l'hétérogénéité des savoirs de l'économie en allant jusqu'à ouvrir les portes à des économistes « purs » : par exemple, « le néo-conservatisme précurseur de l'économiste Dehem » (Fortin, 1984, p. 165). Ces chemins parcourus dans l'espace de confrontation entre la transcendance religieuse et l'universalisme économique ont mis en évidence pour ces chercheurs la normativité de toute connaissance économique (Angers, 1984). Voilà bien un point étrangement commun avec la critique post-moderne des sciences sociales !

Sur ce point fondamental du rôle de la normativité dans la démarche sociologique, il faut bien constater qu'il y a eu peu de progression, oscillant entre des modèles d'attribution d'une idéologie aux chercheurs et la découverte plus récente de l'hétérogénéité du travail de connaissance. Reste le défi que lançait $\mathrm{H}$. Arendt aux sciences sociales, celui d'élaborer une forme de connaissance qui ne soit pas relativiste, qui ne s'évade pas dans les modèles de rationalité fonctionnelle de l'intérêt comme une résorption abrupte du problème de la pluralité des valeurs sociales, qui situe l'action de pensée, la sienne ou celle des autres, à travers l'expérience renouvelée. Pour nous, les travaux sur les médiations sociales de la connaissance contribueront à une sociologie dans la mesure où ils intégreront ces exigences au nouveau cadre de connaissance qu'elles proposent.

\section{RÉSUMÉ}

$\underline{\text { Retour à la table des matières }}$

Cet article traite de l'épistémè de la connaissance économique au Québec à partir des médiateurs et des médiations sociales qui la construisent. L'approche adoptée est celle d'une sociologie de la connaissance à partir du cadre d'analyse des contradictions « fondamentales et flagrantes » proposé par $\mathrm{H}$. Arendt caractérisant la transition à la modernité. À partir de cette approche est reconstruite la localisation sociale des premiers penseurs de l'économie, Édouard Montpetit et Escras Minville, située à travers le savoir religieux issu de leur socialisa- 
tion et le cadre de pensée de l'universalisme économique acquis de par leur éducation. Il est montré que ces activités de connaissance mettent en jeu un travail lié à des publications mais aussi des dispositifs, des réseaux, des revues, travail dont l'analyse permet de comprendre la genèse hétérodoxe de la pensée économique au Québec dans son lieu principal, les HEC de Montréal.

\section{ABSTRACT}

This paper deals with the epistema of economic knowledge in Quebec based on the mediators and social mediations that construct it. The approach adopted is that of a sociology of knowledge based on the analytical frame of the 'fundamental and flagrant' contradictions proposed by $\mathrm{H}$. Arendt characterising the transition to modernity. Based on this approach, the paper reconstructs the social position of the early thinkers of economics, E. Montpetit and E. Minville, stemming from the religious knowledge arising from their socialization and the thought framework of economic universalism acquired through their education. It is shown that these activities of knowledge give rise to work related to publications but also methods, networks, journals, research whose analysis explains the heterodox origin of economic thought in Quebec, mainly centered at Montreal's HEC.

\section{RESUMEN}

Este artículo trata del épistema del conocimiento económico en Quebec a partir de los mediadores y mediaciones sociales que Io construyen. El enfoque adoptado es el de una sociología del conocimiento a partir del marco de análisis de las contradicciones "fundamentales y obvias" propuesto por $\mathrm{H}$. Arendt que caracterizan la transición a la modernidad. A partir de este enfoque se reconstruye la localización social de los primeros perisaclores de la economía, E. Montpetit y E. Minville, situados a través del conocimiento religioso de su socialización y ei marco del pensamiento del universalismo económico adquirido en su educación. Se pone de manifiesto que estas actividades de conocimiento ponen en juego un trabajo vinculado a publicaciones asi como a dispositivos, a redes, a revistas, trabajo cuyo análisis permite incluir la génesis hétérodoxa del pensamiento económico en Quebec hecho en su lugar principal los HEC de Montreal. 


\section{BIBLIOGRAPHIE}

$\underline{\text { Retour à la table des matières }}$

ANGERS, François-Albert, « Témoignages » in Continuité et rupture : les sciences sociales au Québec, G.-H. Lévesque (dir), Montréal, PUM, 1984.

ARENDT, H., La crise de la culture : huit exercices de pensée politique, Paris, Gallimard, 1972.

BEAUD, Michel et Gilles DOSTALER, La pensée économique depuis Keynes : historique et dictionnaire des principaux auteurs, $\mathrm{Pa}-$ ris, Éditions du Seuil, 1993.

BÉDARD, R.J., L'essor économique du Québec, Montréal, Beauchemin, 1969.

BERTHELOT, J.-M., "Les nouveaux défis épistémologiques de la sociologie », Sociologie et sociétés, vol. 30, no 1, 1998, pp. 23-38.

BROCHU, P., Économie coopérative et formes de connaissance. analyse de l'enracinement cognitif au fondement des représentations sociales de l'activité financière dans les caisses d'économie au Québec (1945-1996), thèse de doctorat, Montréal, Université de Montréal, 2002.

CHARBONNEAU, H., Répertoire numérique du fonds de lInstitut d'économie appliquée, Montréal, HEC. DENIS, H. (1980), L'économie de Marx : histoire d'un échec, Paris, PUF, 1991.

DESCOMBES, Vincent, Les institutions du sens, Paris, Éditions de Minuit, 1996. 
DOSTALER, Gilles, "Transition et pensée économique dans l'histoire », Cahiers de recherches sociologiques, vol. 1, no 11, 1988, pp. 19-35.

DUMONT, F., MONTMINY, J.-P. et HAMELIN, J. (dir.) (1971), Idéologies au Canada français, Québec, Presses de l'Université Laval.

Dupuis J-C., Nationalisme et catholicisme, L'Action française de Montréal (1917-1928), Mémoire de maîtrise, Montréal, Université de Montréal, 1992.

ELIAS, N., Engagement et distanciation : contributions à la sociologie de la connaissance, Paris, Fayard, 1993.

FABRE, G., « Un arc transatlantique et sa tagente ou comment se dessine un réseau intellectuel franco-québécois ? "Globe. Revue internationale d'étude québécoise, vol. 7, no 1, 2005, pp. 43-78.

FOISY-GEOFFROY, D., Esdras Minville, le catholicisme social et le nationalisme économique, 1923-1939, Mémoire de maîtrise, Montréal, Université de Montréal, 2001.

FOISY-GEOFFROY, D., Esdras Minville : nationalisme économique et catholicisme social au Québec durant l'entre-deux-guerres, Sillery : Septentrion, 2004.

FORTIN, R., " La recherche économique dans les universités du Québec français : les sources de rupture avec le passé et les défis de l'avenir ", in Continuité et rupture : les sciences sociales au Québec, G.-H. Lévesque (dir.), Montréal, Presses de l'Université de Montréal, 1984, pp. 161-172.

FOURNIER, J. (2005), « Les économistes canadiens-français pendant l'entre-deux-guerres ", Revue d'histoire de l'Amérique française, vol. 58, no 3, 2005, pp. 387-414.

FOURNIER, Marcel, L'entrée dans la modernité : science, culture et société au Québec, Montréal, Éditions Saint-Martin, 1986. [Texte disponible dans Les Classiques des sciences sociales. JMT.] 
GÉRIN-LAJOIE, A., Jean Rivard, le défricheur ; suivi de Jean Rivard, économiste, Saint-Laurent, Québec, BQ, 1993.

GIRBOD, F., « Péguy, Charles (1873-1914) », Universalis 9, édition cédérom, 2003.

GODELIER, Maurice, L'idéel et le matériel : pensée, économies, sociétés, Paris, Fayard, 1984.

GRANGER, G.-G., " Épistémologie économique », in Logique et connaissance scientifique, Jean Piaget (dir), Paris, Encyclopédie de la Pléiade, Gallimard, 1967, p. xii, 1345.

GRANGER, G.-G., « Science, philosophie et idéologie », Raisons pratiques, no 97, 1991, pp. 89-120.

GRANGER, G.-G. (1988), Pour la connaissance philosophique, Paris, O. Jacob, 1988.

GRANOVETTER, M., « Economic Action and Social Structure : The Problem of Embeddedness ", American Journal of Sociology, no 91, novembre 1985, pp. 481-510.

HABERMAS, J., Après Marx, Paris, Fayard, 1985.

HALBWACHS, Maurice, Les cadres sociaux de la mémoire, nouv. éd., Paris, PUF., 1952. [Texte disponible dans Les Classiques des sciences sociales. JMT.]

HALBWACHS, Maurice, La mémoire collective, 2e édition revue et augmentée, Paris, PUF, 1968. [Texte disponible dans Les Classiques des sciences sociales. JMT.]

HARVEY, P., Histoire de l'École des hautes études commerciales, Montréal, Éditions Québec/Amérique, 1994.

HARVEY, P., Histoire de l'École des hautes études commerciales, Montréal, Éditions Québec/Amérique, 2002. 
HEC (1911-1913), «Revue économique canadienne », Montréal.

HENNION, A., La passion musicale : une sociologie de la médiation, Paris, Éditions Métailié, 1993.

HOULE, Gilles, «L'idéologie : un mode de connaissance. Note de recherche », Sociologie et sociétés, vol. 11, no 1, 1979, pp. 123-145. [Texte disponible dans Les Classiques des sciences sociales. JMT.]

HOULE, Gilles, «Famille et politique », Conjonctures, no 3, 1983, pp. 51-61. [Texte disponible dans Les Classiques des sciences sociales. JMT.]

HOULE, Gilles, "L'économie comme forme de connaissance ", Sociologie du Sud-Est, janvier-décembre 1987, pp. 31-48.

HOULE, Gilles, HAMEL, Jacques, SABOURIN, Paul, « Stratégies économiques et développement industriel : l'émergence de Forano », Recherches sociologiques, vol. 25, no 2, 1984, pp. 189-209.

Jacquemin, A., Sélection et pouvoir dans la nouvelle économie industrielle, Puis, Économica, 1985.

LAPLANTE, R., Guyenne, village coopératif. « la petite Russie », Cachan, Éd. de l'ENS-Cachan, 1994.

LEMIRE, M., « Jean Rivard », in Dictionnaire des œuvres littéraires du Québec, M. Lemire (dir.), Montréal, Fides, 1980.

LÉVESQUE, G.-H. (dir.), Continuité et rupture : les sciences sociales au Québec : textes réunis, Montréal, Presses de l'Université de Montréal, 1984.

MINVILLE, Esdras, Invitation à l'étude, Montréal, Fides, 1943.

MINVILLE, Esdras, La forêt, Montréal, Fides, 1944. 
MINVILLE, Esdras, La forêt : étude préparée avec la collaboration de l'École de génie forestier de Québec, Montréal, Fides, 1944.

MINVILLE, Esdras, ANGERS, F.-A. et R. PARADIS, L'économie du Québec et la science économique, Montréal, Fides, Presses HEC, 1979.

MINVILLE, Esdras, ANGERS, F.-A. et R. PARADIS, Systèmes et structures économiques, Montréal, Fides, Presses HEC, 1980.

MINVILLE, Esdras, ANGERS, F.-A. et R. PARADIS, Plan et aménagement. Les secteurs de base, Montréal, Fides, Presses HEC, 1991.

MINVILLE, Esdras, PARADIS, R. et F.-A. ANGERS, Plan et aménagement : les données fondamentales, Montréal, Fides, Presses HEC, 1981.

MINVILLE, Esdras, PARADIS, R. et F.-A. ANGERS, Le travail, Montréal, Fides, Presses HEC, 1982.

MOLINO, J., Pour une histoire de l'interprétation : les étapes de l'herméneutique, Philosophique, vol. 12, no 1, 1985, pp. 75-103.

MONTPETIT, Édouard, «L'indépendance économique du Canada français ", in L'essor économique du Québec, R.-J. Bédard (dir.), Montréal, Beauchemin, 1969, pp. 32-44. [Texte disponible dans Les Classiques des sciences sociales. JMT.]

MONTPETIT, Édouard, Cours d'économie politique, Montréal, HEC, 1921.

MONT PETIT, Édouard, « L'indépendance économique des Canadiens français », L'Action française, vol. 5, no 1, 1921, pp. 32-55.

MONTPETIT, Édouard, La conquête économique, Montréal, Valiquette, 1942. [Texte disponible dans Les Classiques des sciences sociales. JMT.] 
MONTPETIT, Édouard, Souvenirs, Montréal, L'Arbre, 1944.

MUNCK, J. D., L'institution sociale de l'esprit : nouvelles approches de la raison, Paris, PUF, 1999.

PAQUET, G. (1995), « "Le fruit dont l'ombre est la saveur" : réflexions aventureuses sur la pensée économique au Québec », Recherches sociographiques, vol. 26, no 3, 1995, pp. 355-397.

PAQUET, G., « Réflexions iconoscopiques sur la pensée économique au Québec français ", L'Actualité économique, vol. 76, no 1, 2000, pp. 51-66.

PARADIS, R., " Les antécédents de la pensée d'Esdras Minville », in Le travail, F.-A. Angers (dir.), Montréal, Fides, Presses HEC, 1982, pp. 11-36.

RAMOGNINO, N., "Épistémologie, ontologie ou théorie de la description ", in L'enquête ontologique : $d u$ mode d'existence des objets sociaux, P. Livet et R. Ogien (dit.), Paris, Raisons pratiques, 11, Éditions de l'École des hautes études en sciences sociales, 2000, pp. 153-182.

RAMOGNINO, N. et F. GRAO, « Les matérialités sociales et leurs observations. Les leçons de méthode de M. Halbwachs ", Sociologie et sociétés, vol. 29, no 2, 1997, pp. 103-119.

RAYNAULD, André, La propriété des entreprises au Québec les années 60, Montréal, Presses de l'Université de Montréal, 1974.

Rioux, Marcel, Le besoin et le désir : ou, Le code et le symbole : essai. Montréal, L'Hexagone, 1984.

ROSE, M.-C., L'appropriation sociale de l'alimentation au sein d'un projet de mise en marché socialement différenciée, Mémoire de maîtrise, Montréal, Université de Montréal, 2001.

SABOURIN, Paul, " La régionalisation du social : une approche de l'étude de cas en sociologie ", Sociologie et sociétés, vol. 25, no 2, 
1993, pp. 69-82. [Texte disponible dans Les Classiques des sciences sociales. JMT.]

SABOURIN, Paul, «L'économie de la parenté au Québec. Sur la constitution symbolique des formes de circulation matérielle », L'Ethnographie, no 90, 1994, p. 1.

SABOURIN, Paul, «Perspective sur la mémoire sociale de Maurice Halbwachs ", Sociologie et sociétés, vol. 29, no 2, 1997, pp. 139161. [Texte disponible dans Les Classiques des sciences sociales. JMT.]

SOUTHAM, P., « La pensée sociale d'Édouard Montpetit », in Idéologies au Canada français 1930-1939, Fernand Dumont, J-P. Montminy et J. Hamelin (dir.), Québec, Presses de l'Université Laval, 1971, pp. 317-349.

WARREN, J.-P., L'engagement sociologique : la tradition sociologique du Québec francophone, 1886-1955, Montréal, Boréal, 2003.

WARREN, J.-P, La tradition sociologique québécoise francophone (1886-1955) : jalons pour une sociologie de la connaissance, Thèse de doctorat, département de sociologie, Montréal, Université de Montréal, 2001.

Fin du texte 